\title{
Quantifying the Cross-Correlations between Online Market Participation Willingness and Stock Market Dynamics
}

\author{
Gang Chu, ${ }^{1}$ Xiao Li $\left(\mathbb{D},{ }^{2}\right.$ and Yongjie Zhang ${ }^{1}$ \\ ${ }^{1}$ College of Management and Economics, Tianjin University, Tianjin 300072, China \\ ${ }^{2}$ School of Finance, Nankai University, Tianjin 300350, China \\ Correspondence should be addressed to Xiao Li; xiaoli@nankai.edu.cn
}

Received 5 December 2019; Revised 29 April 2020; Accepted 27 May 2020; Published 24 June 2020

Academic Editor: Marcio Eisencraft

Copyright (c) 2020 Gang Chu et al. This is an open access article distributed under the Creative Commons Attribution License, which permits unrestricted use, distribution, and reproduction in any medium, provided the original work is properly cited.

The investors' market participation willingness plays a vital role in the decision-making process of asset allocation. With the newly emerged dataset of investors' market participation willingness, this paper provides the first evidence on the dynamic relationship between market participation willingness and the market dynamics in the Chinese stock market. We select four typical Chinese stock market indices, i.e., SSE50 Index, CSI300 Index, Small and Medium Enterprise Market Index, and Growth Enterprise Market Index, to represent different aspects of the Chinese stock market. Moreover, we use mutual information to measure the overall dependence between market participation willingness and stock market and employ the DCCA cross-correlation coefficient and MF-DCCA to investigate the cross-correlation between market participation willingness and market dynamics. We find that there exist overall dependence and power-law cross-correlation between market participation willingness and the Chinese stock market, and the cross-correlations are significantly multifractal.

\section{Introduction}

Stock market participation is always an important topic in behavioral finance. Investors' market participation willingness directly affects investment decisions on how to allocate their assets to the savings or stock market. High market participation willingness means that more investors participate in the stock market and more capital flows into the stock market. The limited stock market participation is one of the most important market frictions and can help us understand some financial puzzles or anomalies which cannot be explained by frictionless neoclassical models [1]. Existing literature on market participation is more concerned with the determinants of market participation, while little is known about the influence of it on the stock market.

Previous studies have been paying more attention to the determinants of market participation. Some basic determinants of stock market participation have been recognized. The stock market participation willingness is influenced by family wealth [2], investors' financial education [3], IQ [4], gender [5], financial literacy [6], and personality traits [7].
However, in conventional economics of ideal markets and rational decision making, underlying determinants are usually neglected. Liang and Guo [8] highlighted that social interaction plays a significant role in transmitting relevant information to potential investors and found the marginal effect of social interactions on stock market participation. Bonaparte and Kumar [9] found that political activism increases people's propensity to participate in the stock market because their information-gathering costs are lower. Rao et al. [10] focused on the relationship between investor happiness and stock market participation, either directly or indirectly through mutual funds, by using data from China household finance survey (CHFS) and found that happiness affects investors' decisions regarding buying stocks or mutual funds.

As for the influence of market participation. Basak and Cuoco [11] constructed an equilibrium model to study the characterization of equilibrium prices and optimal consumption and investment policies, respectively, and investigated the equilibrium implications of restricted market participation. Guo [12] presents a consumption-based 
model to explain the equity premium puzzle and finds that limited market participation led to lower risk-free rate generating a substantial liquidity premium. Polkovnichenko [13] also studied the implications of limited stock market participation for the equity premium, but he found that limited stock market participation does not play a significant role in explaining the equity premium puzzle. These studies only provide some evidence about the influence of market participation with theoretical models and a serious lack of empirical analysis support. The main reason for this is that the lack of proper data identifying the market participation willingness. Zhang [14] used US country-level racial composition as a proxy for market participation and found that stocks headquartered in countries with a higher percentage of the local white population have more liquidity. However, he only highlighted the influence of local market participation on local stocks, and we do not think the percentage of the white population is an indirect and improper proxy for market participation willingness. It is essential to provide more empirical evidence to explain the influence of market participation on the stock market. In addition, existing studies about market participation all focus on the developed countries' equity market, and the Chinese stock market is neglected. The Chinese stock market is one of the most important parts of the global financial market. Moreover, the Chinese stock market is dominated by individual investors, and their market participation willingness will have a significant impact on market performance. Therefore, the relationship between market participation willingness and the Chinese stock market may be different from that in the developed stock market.

The objective of this paper is to investigate the complex relationship between investors' market participant willingness and the Chinese stock market. Chinese stock market is dominated by individual investors, whose market participation willingness is more likely to affect the stock market. Since the stock market is a far more complex system with fractality properties [15-17], traditional methodologies under the framework of efficiency market hypothesis may not fully explain the relationship between investors' market participation willingness and the stock market. Recently, various methodologies under the theoretical framework of the fractal market hypothesis are put forward to investigate the multifractal property, such as multifractal detrended fluctuation analysis (MF-DFA) [18], multifractal detrended cross-correlation analysis (MF-DCCA) [19-21], and others. In this paper, we employ Yu'e Bao Sentiment Index (YSI) as a proxy for investors' market participation willingness and use the mutual information to measure the overall dependence between the investors' market participation willingness and the stock market; we also employ the DCCA crosscorrelation coefficient and MF-DCCA method to investigate the cross-correlation between investors' market participation willingness and the stock market. Ruan et al. [22] used Yu'e Bao Sentiment Index as proxy for individual investor sentiment and employed the MF-DCCA method to investigate the cross-correlation between investor sentiment and stock market returns. Our paper differs from Ruan et al. [22] in a few key ways. First, the focus of Ruan et al.'s paper [22] is the relationship between investor sentiment and stock returns of Chinese stock market, while we focus on the investors' market participation willingness. Yu'e Bao Sentiment Index has been created to describe changes in users' willingness to enter the market. This index is designed based on data about hundreds of millions of users and millions of transactions, and thus we believe that this index is more appropriate to represent the investors' market participation willingness, rather than investor sentiment. Second, we thoroughly investigate the cross-correlation between investors' market participation willingness and stock market returns, trading volume, and stock market volatility, not just on the stock market return. Third, our study accounts for all major stock indices, including SSE50 Index, CSI300 Index, GEM Index, and SME Index, in Chinese stock market and is therefore generalizable. The SSE50 Index and CSI300 Index represent the mature and large-capitalization listed firms, which is not easy to be affected by market participation willingness. However, the GEM Index and SME Index represent the growing and small-capitalization listed firms, which is easier to be affected by market participation willingness. Thus, the dynamic relationship can be different when considering different types of stocks.

Our paper contributes to a growing literature in the following aspects. First, we focus on the relationship between investors' market participation willingness and the stock market. Existing literature about behavioral finance mainly focused on investor happiness [23, 24], investor sentiment [25-27], investor attention [28-30], and geographical information [31, 32]. This paper is the first one to give empirical analysis on the nonlinear cross-correlation between investors' market participation willingness and Chinese stock market performance. Second, we employ YSI as the proxy for market participation willingness in Chinese stock market. YSI is the newly emerged dataset on investors' willingness to participate in the stock market, which directly describes the market participation willingness based on the daily transaction behavior and data of over 200 million Yu'e Bao users. Third, we combine mutual information analysis and MF-DCCA analysis to examine the nonlinear relationship between investors' market participation willingness and Chinese stock performance. This new empirical perspective expands the existing literature.

The rest of this paper is organized as follows. Section 2 describes the data and preliminary test statistics. Section 3 introduces the methodology of mutual information and MFDCCA. Section 4 represents the empirical results, and Section 5 makes a conclusion.

\section{Data and Descriptive Statistics}

This paper mainly uses the daily Yu'e Bao Sentiment Index (YSI) as the proxy for market participation willingness, which is derived from the TianHong Fund official website (http://www.thfund.com.cn/yuebao/emotions). Yu'e Bao is China's first-ever Internet fund specially designed for Alipay. It amasses roughly RMB 1.13 trillion in assets and 588 million users under management to 2019, marking it the largest money market fund in China. The YSI is constructed 
by TianHong Fund by mining the fund data of Yu'e Bao users. This index is based on 200 million users and 10 billion pieces of data of the transaction, using big data analysis models and cloud computing technology to reflect the changes in users' willingness to enter the stock market. There is no doubt that Yu'e Bao Sentiment Index is an objective and accurate proxy for portraying investor's participation willingness in China. Meanwhile, compared with other market participation proxies, YSI can provide daily data excluding the IPO (Initial Public Offering) data. The time span of this index is from April 1, 2014, to May 10, 2018. We calculate the daily abnormal investor sentiment index (Ab_YSI) as the daily raw YSI as provided by TianHong Fund minus the average raw YSI over the prior 30 trading days, scaled by the average raw YSI over the prior 30 trading days. The detailed definition is as follows:

$$
\mathrm{Ab}_{-} \mathrm{YSI}_{t}=\frac{\mathrm{YSI}_{t}}{(1 / 30) \sum_{k=1}^{30} \mathrm{YSI}_{i-k}}-1,
$$

where YSI $_{t}$ is the Yu'e Bao Sentiment Index in day $t$.

To study the relationship between market participation willingness and stock market, we choose four major stock market indices, including SSE50 Index, CIS300 Index, SSE SME Composite (SME), and Growth Enterprise Market (GEM) Index. SSE50 and CSI300 indices are composed of listed companies with large market value and high liquidity, SME Index is composed of small size companies, and GEM Index is composed of high risk and rapid growth companies. We get the stock market index data from CSMAR (CSMAR financial dataset, China Stock Market Accounting Research financial dataset) financial database in the same sample period from April 1, 2014, to May 10, 2018. Formally, we estimate daily market returns and abnormal trading volume. We calculate daily abnormal volume as daily trading volume minus the average trading volume for a 30-trading-day period, divided by the average trading volume for a 30trading-day period. The detailed definitions are as follows:

$$
\text { Return }_{t}=\log \left(\frac{P_{t}}{P_{t-1}}\right) \text {, }
$$

where $P_{t}$ is the stock market index return in day $t$.

$$
\mathrm{Ab}_{-} \text {Volume }_{t}=\frac{\text { Volume }_{t}}{(1 / 30) \sum_{k=1}^{30} \text { Volume }_{i-k}}-1,
$$

where Volume ${ }_{t}$ is the stock market trading volume in day $t$.

The variance of returns can be predicted using particular time series models, and the Generalized Autoregressive Conditional Heteroscedasticity (GARCH) seems to be the most successful $[33,34]$. We use GARCH $(1,1)$ to calculate the daily stock market returns volatility. The GARCH $(1,1)$ model is as follows:

$$
\begin{aligned}
& \varepsilon_{t}=\sqrt{h_{t}} v_{t}, \\
& h_{t}=\alpha_{0}+\beta_{1} h_{t-1}+\alpha_{1} \varepsilon_{t-1}^{2},
\end{aligned}
$$

where $h_{t}$ is the conditional variance.
Figure 1 illustrates the daily time series YSI, SSE50 Index, CSI300 Index, SME Index, and GEM Index. As shown in Figure 1, the trend of YSI is similar to Chinese stock market indices.

Table 1 reports the descriptive statistics for YSI, daily stock market returns, abnormal trading volume, and returns volatility, including the mean, standard deviation, skewness, kurtosis, minimum, and maximum. As shown in the table, YSI ranges from 415.1 to 2743.41 , and the mean is 1033.156 . The abnormal YSI varies from a minimum of -0.563 to a maximum of 1.284 .

\section{Methodology}

In this section, we discuss the main methods used in this paper, including mutual information (MI), DCCA crosscorrelation coefficient, and multifractal detrended crosscorrelation analysis (MF-DCCA).

3.1. Mutual Information. In information theory, the mutual information (MI) is a measure of the mutual dependence between the two time series $[35,36]$. Specifically, MI quantifies the random dependency between two stochastic variables without making any hypothesis about the nature of the relationship [37]. To measure the correlation between two equal length time series $X=\left\{x_{t}\right\}$ and $Y=\left\{y_{t}\right\}$, $t=1,2,3, \ldots, N$, we compute the Shannon entropy $H(X)$ (or $H(Y)$ ) as follows:

$$
H(X)=-\sum_{x \in X} p_{X}(x) \log p_{X}(x),
$$

where $p_{X}(x)$ (or $\left.p_{Y}(y)\right)$ is the probability density function of a random variable $X$ (or $Y$ ).

For a bivariate case, the Shannon joint entropy is calculated as follows:

$$
H(X ; Y)=-\sum_{x \in X} \sum_{y \in Y} p_{X, Y}(x, y) \log p_{X, Y}(x, y),
$$

where $p_{X, Y}(x, y)$ is the joint probability density function of two random variables $X$ and $Y$.

If the random variable $Y$ depends on the random variable $X$, conditional entropy can be used to measure the remaining uncertainly in $X$ with given $Y$. The conditional entropy is defined as follows:

$$
H(X \mid Y)=-\sum_{x \in X} \sum_{y \in Y} p_{X, Y}(x, y) \log p_{X, Y}(x \mid y) .
$$

Generally, the conditional entropy $H(X \mid Y)$ can also be presented in terms of joint entropy $H(X, Y)$.

$$
H(X \mid Y)=H(X ; Y)-H(Y) .
$$

To quantify the dependence between variables $X$ and $Y$, the mutual information is wieldy used. The mutual information of two random variables $X$ and $Y$ is defined as follows: 


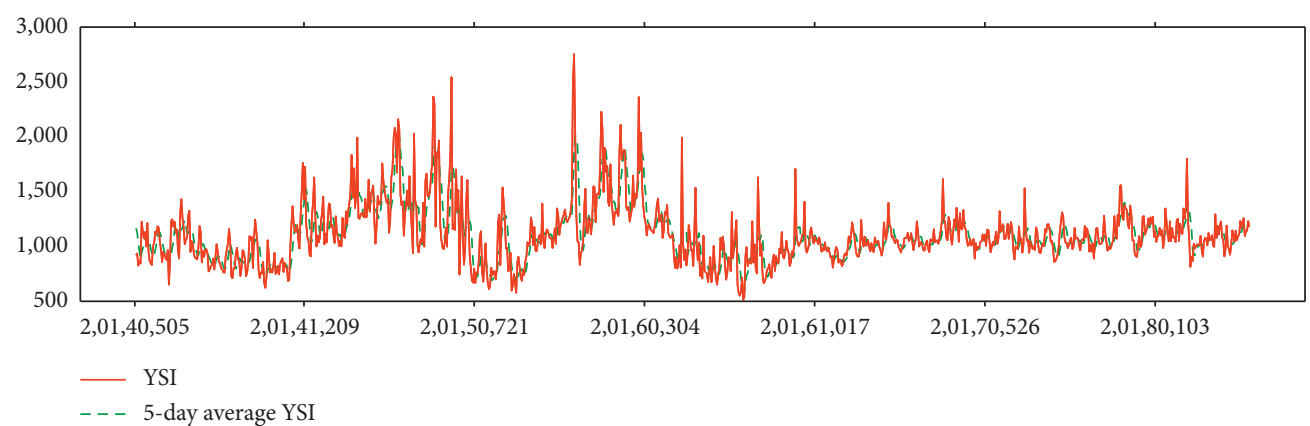

(a)

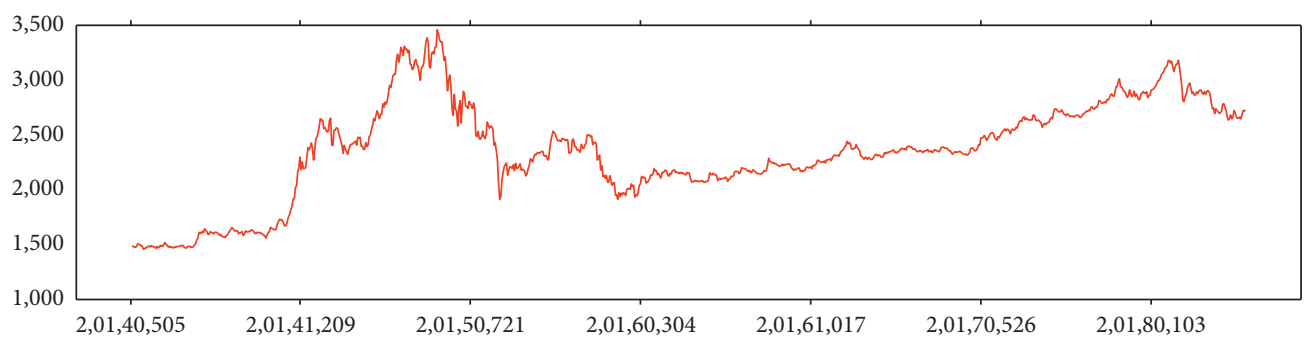

(b)

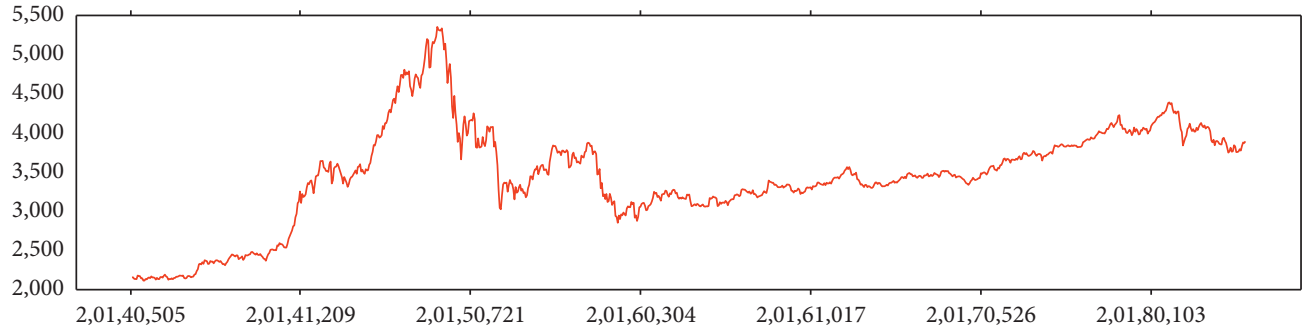

(c)

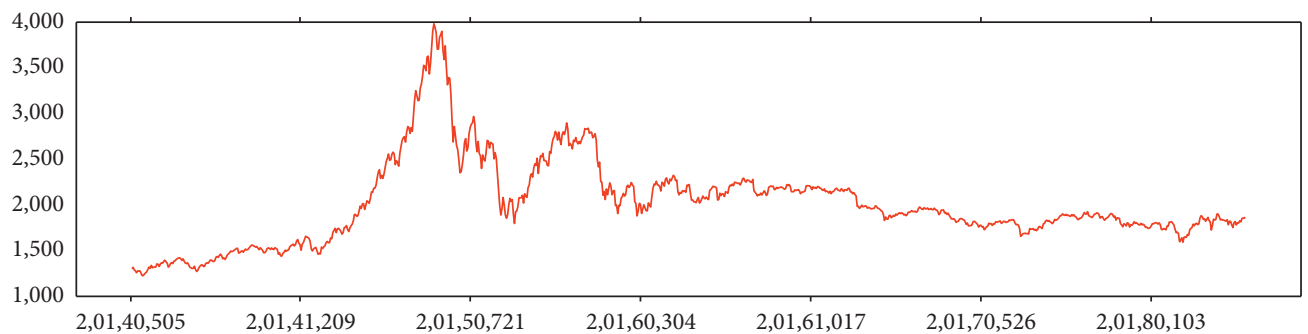

(d)

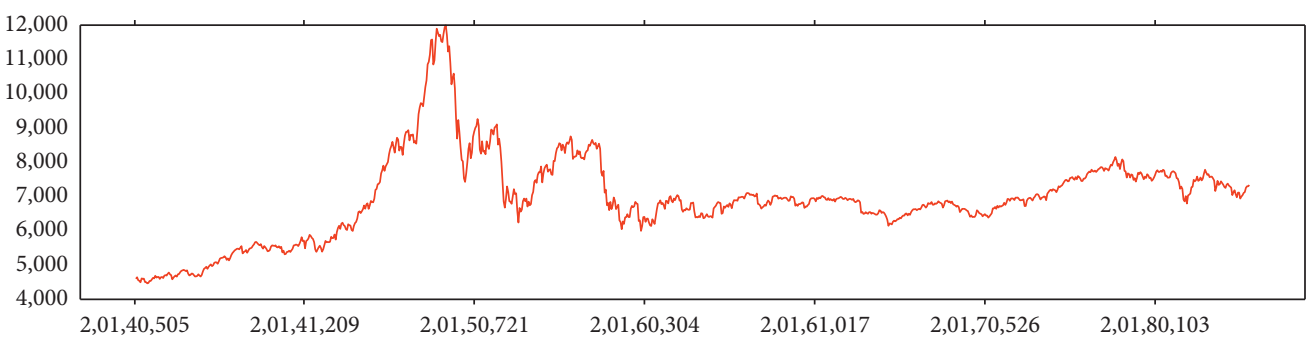

(e)

Figure 1: Time series of (a) YSI, (b) SSE50, (c) CSI300, (d) GEM, and (e) SME from May 1, 2014, to May 10, 2018.

$$
\operatorname{MI}(X, Y)=\sum_{x \in X} \sum_{y \in Y} p_{X, Y}(x, y) \log \left(\frac{p_{X, Y}(x, y)}{p_{X}(x) p_{Y}(y)}\right) .
$$

Combining with Shannon's entropy, the mutual information $\operatorname{MI}(X, Y)$ can be expressed as

$$
\operatorname{MI}(X, Y)=H(X)-H(X \mid Y) \text {. }
$$


TABLE 1: Summary statistics.

\begin{tabular}{|c|c|c|c|c|c|c|}
\hline & Mean & Std. dev. & Skew. & Kurt. & Max & Min \\
\hline \multicolumn{7}{|c|}{ (A) Yu'e Bao Sentiment Index } \\
\hline YSI & 1033.156 & 290.028 & 1.555 & 7.505 & 2743.41 & 415.1 \\
\hline Ab_YSI & 0.00754 & 0.0457 & 1.169 & 7.064 & 1.284 & -0.563 \\
\hline \multicolumn{7}{|c|}{ (B) Chinese stock market return } \\
\hline SSE50 & 0.000266 & 0.00709 & -0.839 & 10.336 & 0.0328 & -0.0428 \\
\hline CSI300 & 0.000261 & 0.00691 & -1.189 & 10.062 & 0.0282 & -0.0398 \\
\hline SME & 0.000211 & 0.00789 & -1.042 & 7.507 & 0.0287 & -0.0382 \\
\hline GEM & 0.000165 & 0.00934 & -0.669 & 5.835 & 0.0301 & -0.0405 \\
\hline \multicolumn{7}{|c|}{ (C) Chinese stock market trade volume } \\
\hline SSE50 & 0.0339 & 0.461 & 1.521 & 6.315 & 2.528 & -0.752 \\
\hline CSI300 & 0.0254 & 0.351 & 1.189 & 5.257 & 1.968 & -0.705 \\
\hline SME & 0.0222 & 0.219 & 0.407 & 3.462 & 0.931 & -0.758 \\
\hline GEM & 0.0358 & 0.232 & 0.448 & 3.343 & 0.819 & -0.767 \\
\hline \multicolumn{7}{|c|}{ (D) Chinese stock market volatility } \\
\hline SSE50 & 0.000276 & 0.000362 & 2.506 & 9.999 & 0.00221 & 0.0000259 \\
\hline CSI300 & 0.000261 & 0.000343 & 2.441 & 9.240 & 0.00195 & 0.0000249 \\
\hline SME & 0.000321 & 0.000331 & 1.819 & 5.489 & 0.00152 & 0.0000605 \\
\hline GEM & 0.0004502 & 0.000439 & 1.659 & 4.928 & 0.00204 & 0.0000908 \\
\hline
\end{tabular}

This table reports the mean, standard deviation (Std. dev.), skewness (Skew.), kurtosis (Kurt.), minimum (Min), and maximum (Max) of Yu'e Bao Sentiment Index (YSI and Ab_YSI), the return, volume, and volatility for four stock market indices: SSE50, CSI300, SME, and GEM. The sample period extends from May 5, 2014, through May 10, 2018.

By substituting equations (7) and (8), $\mathrm{MI}(X, Y)$ can be rewritten as

$$
\operatorname{MI}(X, Y)=H(X)+H(Y)-H(X ; Y) .
$$

Therefore, we can calculate mutual information between two random variables by estimating probability density functions $p_{X, Y}(x, y)$. In this paper, the probability density function is estimated by the method of equal distance histogram, which was proposed by Scott [38] to describe a onedimensional or two-dimensional probability density function.

In equation (11), we have $0 \leq \mathrm{MI}(X, Y) \leq \infty$ [39]. We follow Granger and Lin [40] to use a standard measure for mutual information, which is defined as

$$
I(X, Y)=\sqrt{1-e^{-2 \mathrm{MI}(X, Y)}} .
$$

This measure ranges from 0 to 1 and can directly comparable with the linear correlation coefficient. Compared with traditional correlation coefficients, this function $I(X, Y)$ can capture the overall dependence, both linear and nonlinear, between two random variables.

\subsection{Multifractal Detrended Cross-Correlation Analysis} (MF-DCCA). The detrended fluctuation analysis (DFA) was proposed by Peng et al. [18] to explore the long-range correlations and multifractal features of a single nonstationary time series. Later, Kantelhard et al. [41] extended the DFA to multifractal system and proposed multifractal detrended fluctuation analysis (MF-DFA) to investigate the long-range auto-correlations of the financial time series.
Podobnik and Stanley [42] proposed a generalization of the DFA called detrended cross-correlation analysis (DCCA) method to explore the long range between two nonstationary time series. Based on MF-DFA and DCCA, Zhou [43] proposed multifractal detrended cross-correlation analysis (MF-DCCA) to detect the multifractal features of two nonstationary time series.

In this study, we adopt MF-DCCA to analyze the dynamic relationship between market participation willingness measured by YSI and Chinese stock market. Next, we will give a brief introduction to the methodology of MF-DCCA.

Consider any two equal length time series $\left\{x_{i}\right\}$ and $\left\{y_{i}\right\}$, $i=1,2,3, \ldots, N$; the detailed steps of the MF-DCCA can be summarized as follows:

Step 1. Construct the profile of time series.

$$
X_{i}=\sum_{k=1}^{i}\left(x_{k}-\bar{x}\right), Y_{i}=\sum_{k=1}^{i}\left(y_{k}-\bar{y}\right)
$$

where $\bar{x}=(1 / N) \sum_{k=1}^{N} x_{k}$ and $\bar{y}=(1 / N) \sum_{k=1}^{N} y_{k}$.

Step 2. We divide the two profiles $X_{i}$ and $Y_{i}$ into $N_{s}=$ $\operatorname{int}[N / S]$ nonoverlapping segments with the same length $s$. Consider that the length of the time series $(N)$ is not always an integral multiple of the time scale $s$, and a short part segment at the end of each profile may remain. To make sure more information is contained in the time series, the same divided procedure is repeated starting from the end of each profile. So, we will get $2 \mathrm{~N}_{s}$ nonoverlapping segments. In this paper, we set the scale $s$ as $10<s<N / 4$.

Step 3. For each segment, we calculate the local trends by least-square fit of the series. 


$$
\begin{aligned}
& \widetilde{X}_{\lambda}(j)=\delta_{k} j^{m}+\cdots+\delta_{1} j+\delta_{0}, \\
& \widetilde{Y}_{\lambda}(j)=\gamma_{k} j^{m}+\cdots+\gamma_{1} j+\gamma_{0},
\end{aligned}
$$

where

$j=1,2, \ldots ; \lambda=1,2,3, \ldots, 2 N_{s} ; m=1,2,3, \ldots$.

Step 4. Calculate the detrended variances for each segment depending on the segment $\lambda$.

$F^{2}(\lambda, s)=\frac{1}{s} \sum_{j=1}^{s}\left[X_{(\lambda-1) s+j}-\tilde{X}_{(\lambda-1) s+j}\right]\left[Y_{(\lambda-1) s+j}-\tilde{Y}_{(\lambda-1) s+j}\right]$,

where $\lambda=1,2, \ldots, N_{s} ; \widetilde{X}$ and $\widetilde{Y}$ are the local trends.

$$
\begin{aligned}
F^{2}(\lambda, s)= & \frac{1}{s} \sum_{j=1}^{s}\left[X_{N-\left(\lambda-N_{s}\right) s+j}-\widetilde{X}_{N-\left(\lambda-N_{s}\right) s+j}\right] \\
& \cdot\left[Y_{N-\left(\lambda-N_{s}\right) s+j}-\widetilde{Y}_{N-\left(\lambda-N_{s}\right) s+j}\right],
\end{aligned}
$$

where $\lambda=N_{s}+1, N_{s}+2, \ldots, 2 N_{s} ; \widetilde{X}$ and $\widetilde{Y}$ are the local trends.

Step 5. Take the mean of the local trends of all detrended segments, and we get the qth-order fluctuation function:

$$
F_{q}(s)=\left\{\frac{1}{2 N_{s}} \sum_{\lambda=1}^{2 N_{s}} F^{2}(s, \lambda)^{q / 2}\right\}^{1 / q}
$$

and for $q=0$, this function can be defined as

$$
F_{0}(s)=\exp \left\{\frac{1}{4 N_{s}} \sum_{\lambda=1}^{2 N_{s}} \ln \left[F^{2}(s, \lambda)\right]\right\} \text {. }
$$

Step 6. By observing the plot of log-log plots of $F_{q}(s)$, we can analyze the scaling behavior of the fluctuations. If the two series are long-range cross-correlated, there is power-law relationship between them as follows:

$$
F_{q}(s) \propto s^{h_{x y}(q)} .
$$

This is equivalent to

$$
\log F_{q}(s)=h_{x y}(q) \log (s)+\log C,
$$

where $h_{x y}(q)$ denotes the slope of log-log plots of $F_{q}(s)$ versus $s$, which is computed by the method of OLS. And the value of $h_{x y}(q)$ indicates the crosscorrelation of the two time series. In this paper, $q$ varies from -10 to 10 . If $q>0, h_{x y}(q)$ reveals the scaling behaviors of the segments with large fluctuations; if $q<0, h_{x y}(q)$ reveals the scaling behaviors of the segments with minor fluctuations; especially, when $q=2$, the scaling exponent $h_{x y}(q)$ is the generalized Hurst exponent, and MF-DCCA reduces to DCCA. When $X(i)$ and $Y(i)$ denote the same time series, MF-DCCA reduces to MF-DFA.
Additionally, when $h_{x y}(q)>0.5$, the cross-correlation between the two time series is persistent (positive), indicating that rise in one series is statistically likely to be followed by the increase of another; when $h_{x y}(q)<0.5$, the cross-correlation between the two time series is antipersistent (negative), indicating that rise in one series is statistically likely to be followed by the decrease in another; and when $h_{x y}(q)=0.5$, there are no cross-correlations between the two time series and the time series can be described as random walk. Step 7. Following Shadkhoo and Jafari [44], we compute the multifractal scaling exponent $\tau_{x y}(q)$, called the Renyi exponent, to describe the multifractal property.

$$
\tau_{x y}(q)=q h_{x y}(q)-1
$$

If Renyi exponent $\tau_{x y}(q)$ is linear function of $q$, the cross-correlations of two series are monofractal. Otherwise, the relationships are multifractal.

Step 8. Through Legendre transform, we can obtain the multifractal spectrum $f(\alpha)$ as follows:

$$
\alpha_{x y}(q)=H_{x y}(q)+q H_{x y}^{\prime}(q)
$$

and

$$
f_{x y}(\alpha)=q\left[\alpha_{x y}-H_{x y}(q)\right]+1,
$$

where $\alpha_{x y}$ shows the degree of singularity of the time series and $f_{x y}(\alpha)$ reflects the fractal dimension of $\alpha$. Step 9. Calculate the multifractal intensity $\Delta H$.

$$
\Delta H=H_{\max }(q)-H_{\min }(q),
$$

where the larger $\Delta H$ is, the higher the degree of multifractality is.

3.3. DCCA Cross-Correlation Coefficient. Zebende [45] proposed a new coefficient, namely, DCCA cross-correlation coefficient, to quantify the level of cross-correlation between nonstationary time series [46, 47]. The DCCA cross-correlation coefficient is defined in terms of the DFA method and the DCCA method, which is the ratio of the detrended covariance's function $F_{\text {DCCA }}^{2}$ to the product of two detrended variance function $F_{\mathrm{DFA}}$. This cross-correlation coefficient is widely used to measure the relations between two time series at different scales [48]. The function is defined as follows:

$$
\rho_{\text {DCCA }}(S)=\frac{F_{x y}^{2}(s)}{F_{x x}(s) F_{y y}(s)},
$$

where $F_{x y}^{2}(s)$ is the mean of the detrended covariance function. The functions $F_{x x}$ and $F_{y y}$ are, respectively, the root mean square fluctuation of each time series $\left\{x_{t}\right\}$ and $\left\{x_{t}\right\}$ separately. $\rho_{\text {DCCA }}$ ranges from -1 to 1 . A value of $\rho_{\text {DCCA }}=0$ means there is no cross-correlation. When $\rho_{\text {DCCA }}=1$, the two time series are completely antipersistent and positively correlated. When $\rho_{\text {DCCA }}=-1$, the two time series are completely negatively correlated. 


\section{Empirical Results}

4.1. Mutual Information Analysis. We start our analysis by investigating the overall dependence between the market participation willingness, measured by YSI, and Chinese stock market. Table 2 reports the mutual information $(I(X, Y))$ between market participation willingness and stock market returns, trading volume, and returns volatility, respectively. As shown in Table $2, I(X, Y)$ between market participation willingness and Chinese stock market returns are all beyond 0.88 . The mutual information between market participation willingness and Chinese stock market volume is beyond 0.89 and that between market participation willingness and Chinese stock market returns volatility is beyond 0.87 . These results suggest that the market participation willingness contains much more dynamic information about Chinese stock market.

4.2. Cross-Correlation Test. To examine whether there is cross-correlation between market participation willingness and Chinese stock market, we refer to a cross-correlation test index $Q_{c c}(m)$ proposed by Podobnik et al. [49]. $Q_{c c}(m)$ is defined as

$$
Q_{\mathrm{cc}}(m)=N^{2} \sum_{i=1}^{m} \frac{A_{i}^{2}}{N-i},
$$

where the cross-correlation function $A_{i}^{2}$ is given by

$$
A_{i}^{2}=\frac{\sum_{k=i+1}^{N} x_{k} y_{k-1}}{\sqrt{\sum_{k=1}^{N} x_{k}^{2}} \sum_{k=1}^{N} y_{k}^{2}},
$$

where $\left\{x_{t}, t=1,2, \ldots, N\right\}$ and $\left\{y_{t}, t=1,2, \ldots, N\right\}$ are two time series.

The cross-correlation statistic $Q_{c c}(m)$ is approximately followed by $\chi^{2}(m)$ distributed with $m$ degrees of freedom. The null hypothesis of cross-correlation is that there exists no cross-correlation between two time series. We set the critical values for the $\chi^{2}(\mathrm{~m})$ distribution at 5\% significance level for different degrees of freedom $m$. If the value of $Q_{c c}(m)$ exceeds the critical value of $\chi^{2}(m)$ distribution, the two time series have significant cross-correlations. Otherwise, no cross-correlations exist.

According to equations (26) and (27), we calculate the cross-correlation statistic $Q_{c c}(m)$ for the two time series with the degree of freedom varying from 1 to 800 . The test results of market participation willingness and Chinese stock market returns are presented in Figure 2. The solid lines denote the critical value of $\chi^{2}(m)$ at $5 \%$ significance level. The dashed lines represent the value of $Q_{c c}(m)$. The crosscorrelation statistic $Q_{\mathrm{cc}}(m)$ for four market indices' returns, SSE50, CIS300, SME, and GEM, and market participation willingness is larger than the critical value for the $\chi^{2}$ distribution at $5 \%$ level of significance, which means the Chinese stock market returns and market participation willingness are strongly cross-correlated. Figure 3 illustrates the cross-correlation statistic $Q_{c c}(m)$ for Chinese stock market volume and market participation willingness. The dashed lines in Figure 3 indicate that there is a long-range cross-correlation between Chinese stock market volume and market participation willingness. And we can also find that there exists a long-range cross-correlation between stock market returns volatility and market participation willingness in Figure 4.

4.3. DCCA Cross-Correlation Coefficient Analysis. The DCCA cross-correlation coefficient is quite different from the most popular measurement of the Pearson correlation coefficient and Spearman correlation coefficient, as these two measurements require the time-series to be stationary. However, the DCCA coefficient can measure the correlations between two nonstationary time series at different time scales. In this paper, we intend to apply the DCCA crosscorrelation coefficient to investigate the cross-correlations between investors' market participation willingness (YSI) and stock market performance. Based on the different window size values $s \quad(n=10,50,100,150,200,250$, $300,350,400)$, Table 3 presents calculation results for DCCA cross-correlation coefficient $\left(\rho_{\text {DCCA }}\right)$. Our results show that all correlation coefficients are positive, meaning that investors' market participation willingness has positive correlation levels with the stock market, including stock returns, stock trading volume, and stock volatility. The results presented in Table 3 show that all of the $\rho_{\text {DCCA }}$ fall within the interval at $[0.4,1]$, further verifying the crosscorrelation test results.

4.4. Multifractal Detrended Cross-Correlation Analysis. As we show that there is serious long-range cross-correlation between market participation willingness and Chinese stock market, we can use the multifractal detrended cross-correlation analysis (MF-DCCA) to further investigate the cross-correlation between market participation willingness, measured by YSI, and Chinese stock market returns and trading volume, respectively.

Figure 5 illustrates the log-log plots of $F_{x y q}(s)$ versus $s$ for market participation willingness and four major Chinese stock market indices' returns; the order $q$ of lines decreases from top to bottom. As shown in Figure 4, for all series pairs, $F_{x y q}(s)$ increases linearly along with the scales $s$, implying that power-law behavior and long-range cross-correlations exist between market participation willingness and Chinese stock market returns. Additionally, Figure 6 illustrates the log-log plots for market participation willingness and four major Chinese stock market indices' trading volume, and Figure 7 illustrates the log-log plots for Chinese stock market returns volatility and market participation willingness. The linear log-log lines in Figures 6 and 7 both prove the existence of the power-law behavior and cross-correlations between market participation willingness and Chinese stock market trading volume and returns volatility, respectively.

Furthermore, we compute cross-correlation generalized Hurst exponents $\left(h_{x y}(q)\right)$ based on equations (18)-(27). Figure 8 illustrates the cross-correlation generalized Hurst exponents between market participation willingness and Chinese stock market returns varying with $q$. As we can see, the cross-correlation generalized Hurst exponents are 
TABLE 2: The mutual information between Chinese stock market and market participation willingness.

\begin{tabular}{lccc}
\hline Market index & YSI and return & YSI and Ab_Volume & YSI and volatility \\
\hline SSE50 & $0.884^{* * *}$ & $0.894^{* * *}$ & $0.879^{* * *}$ \\
CSI300 & $0.885^{* * *}$ & $0.893^{* * *}$ & $0.879^{* * *}$ \\
SME & $0.891^{* * *}$ & $0.896^{* * *}$ & $0.889^{* * *}$ \\
GEM & $0.893^{* * *}$ & $0.898^{* * *}$ & $0.890^{* * *}$ \\
\hline
\end{tabular}

This table reports the mutual information between Chinese stock market and YSI.

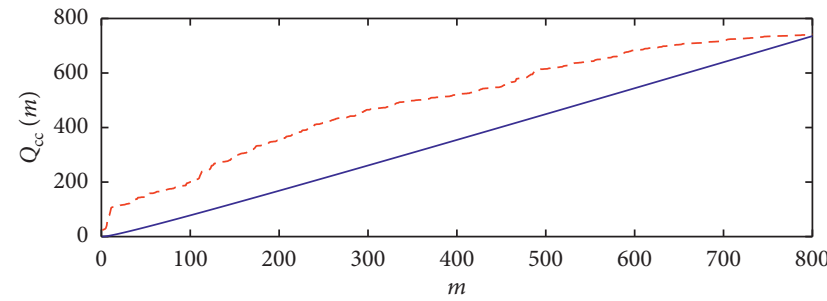

- Critical value

- - - SME-YSI

(a)

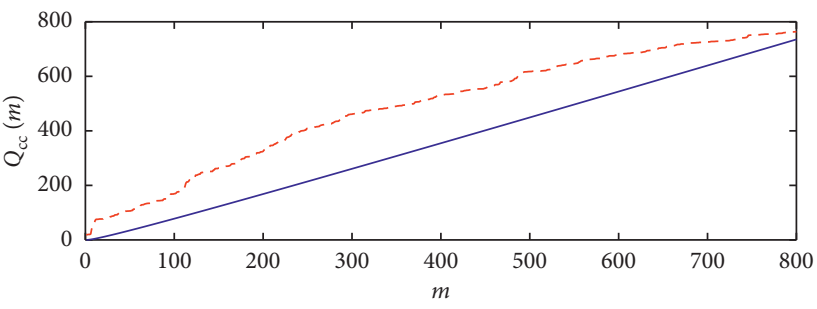

_- Critical value

- . - CSI300-YSI

(c)

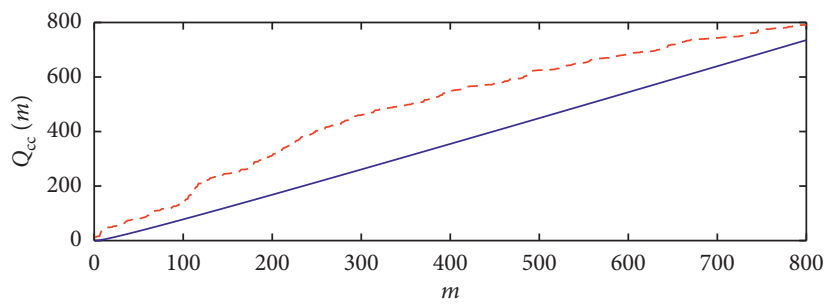

- Critical value

_ - - SSE50-YSI

(b)

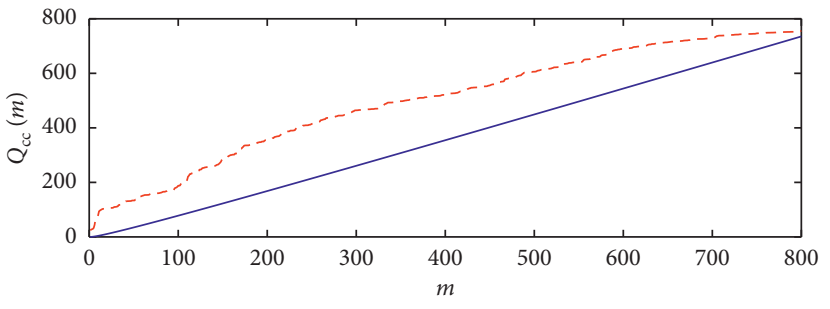

Critical value

- . - GEM-YSI

FIgURE 2: The cross-correlation statistic $Q_{c c}(m)$ for market participation willingness and Chinese stock market returns.

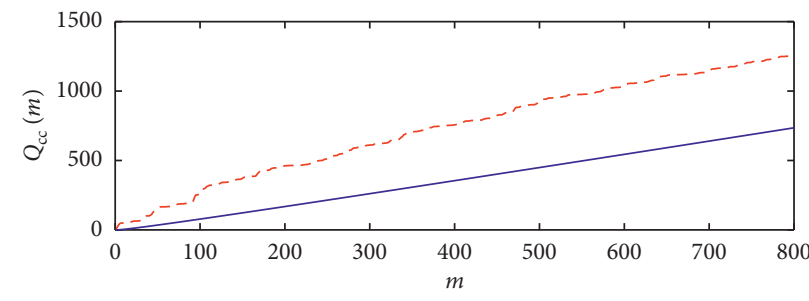

Critical value

- - SME-YSI

(a)

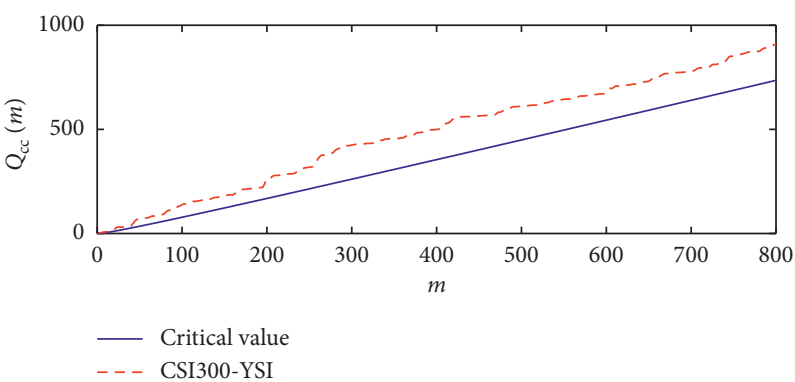

(c)

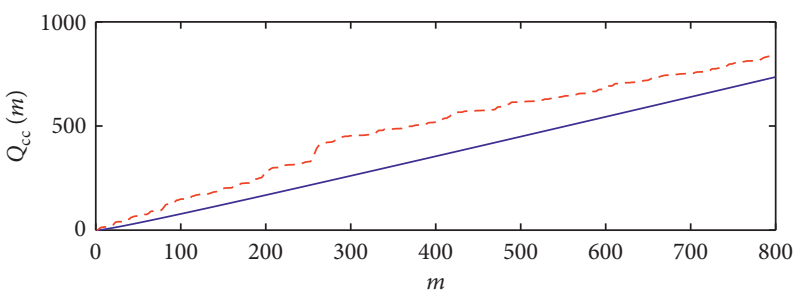

Critical value

_. - SSE50-YSI

(b)

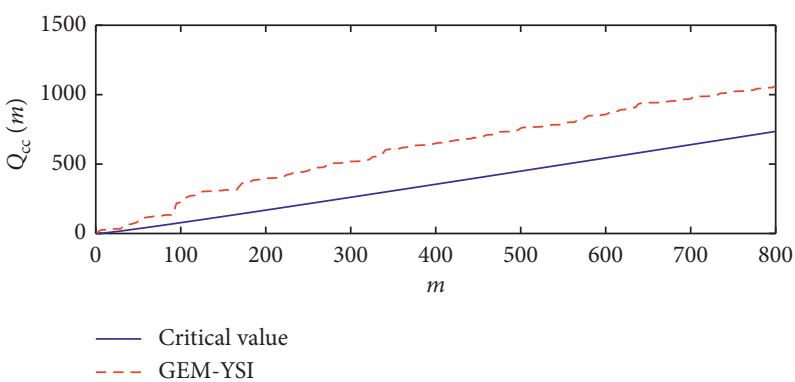

(d)

Figure 3: The cross-correlation statistic $Q_{c c}(m)$ for market participation willingness and Chinese stock market volume. 


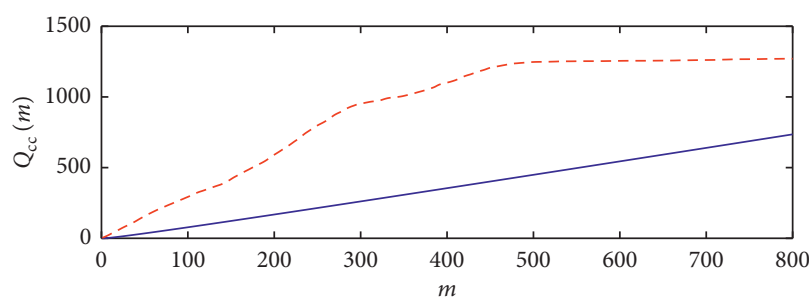

_ Critical value

- - SME-YSI

(a)

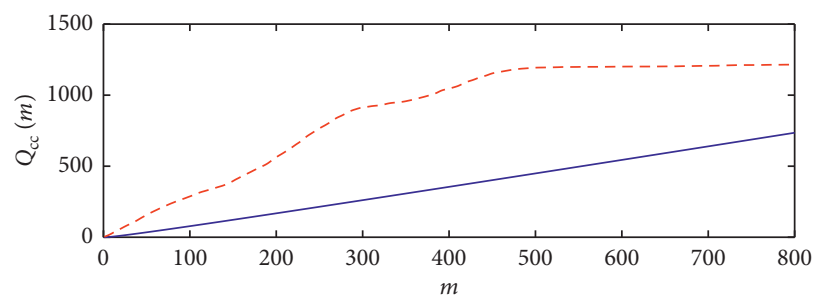

- Critical value

- - - CSI300-YSI

(c)

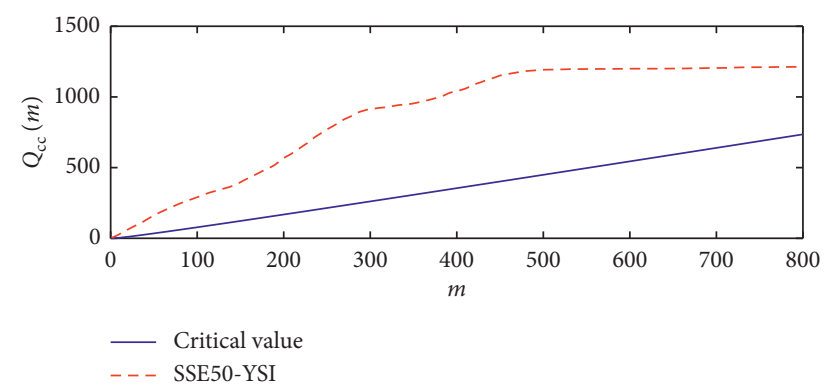

(b)

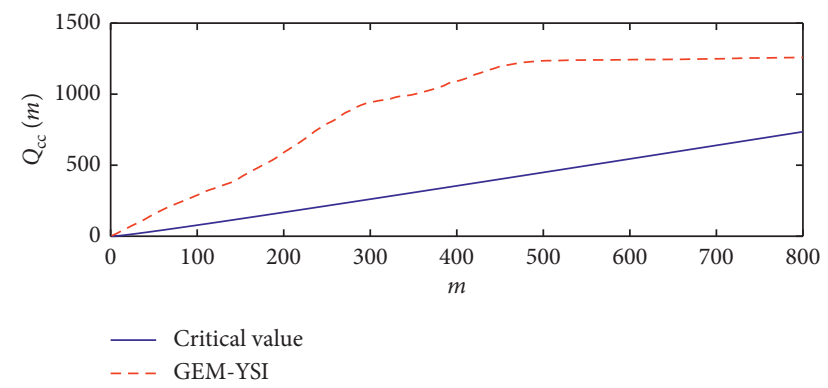

(d)

FIgURE 4: The cross-correlation statistic $Q_{\mathrm{cc}}(m)$ for market participation willingness and Chinese stock market returns volatility.

TABle 3: The detrended cross-correlation coefficient ( $\rho$ (DCCA)) for a given window size $s$.

\begin{tabular}{|c|c|c|c|c|c|c|c|c|c|}
\hline Size (s) & 10 & 50 & 100 & 150 & 200 & 250 & 300 & 350 & 400 \\
\hline \multicolumn{10}{|c|}{ (A) The detrended cross-correlation coefficient between YSI and market returns } \\
\hline SSE50 & 0.618 & 0.630 & 0.657 & 0.652 & 0.649 & 0.673 & 0.677 & 0.639 & 0.634 \\
\hline CSI300 & 0.651 & 0.652 & 0.673 & 0.666 & 0.654 & 0.676 & 0.681 & 0.648 & 0.662 \\
\hline SME & 0.671 & 0.670 & 0.698 & 0.668 & 0.646 & 0.642 & 0.637 & 0.615 & 0.663 \\
\hline GEM & 0.661 & 0.682 & 0.693 & 0.672 & 0.659 & 0.661 & 0.660 & 0.632 & 0.658 \\
\hline \multicolumn{10}{|c|}{ (B) The detrended cross-correlation coefficient between YSI and market volume } \\
\hline Size (s) & 10 & 50 & 100 & 150 & 200 & 250 & 300 & 350 & 400 \\
\hline SSE50 & 0.548 & 0.574 & 0.544 & 0.567 & 0.557 & 0.574 & 0.584 & 0.637 & 0.589 \\
\hline CSI300 & 0.568 & 0.593 & 0.559 & 0.559 & 0.564 & 0.570 & 0.581 & 0.617 & 0.599 \\
\hline SME & 0.619 & 0.608 & 0.621 & 0.615 & 0.638 & 0.642 & 0.648 & 0.627 & 0.656 \\
\hline GEM & 0.599 & 0.614 & 0.614 & 0.605 & 0.610 & 0.644 & 0.629 & 0.631 & 0.647 \\
\hline \multicolumn{10}{|c|}{ (C) The detrended cross-correlation coefficient between YSI and market volatility } \\
\hline Size (s) & 10 & 50 & 100 & 150 & 200 & 250 & 300 & 350 & 400 \\
\hline SSE50 & 0.414 & 0.519 & 0.495 & 0.473 & 0.552 & 0.578 & 0.598 & 0.617 & 0.587 \\
\hline CSI300 & 0.424 & 0.518 & 0.506 & 0.529 & 0.556 & 0.559 & 0.586 & 0.648 & 0.558 \\
\hline SME & 0.433 & 0.507 & 0.524 & 0.551 & 0.576 & 0.556 & 0.572 & 0.586 & 0.550 \\
\hline GEM & 0.495 & 0.541 & 0.565 & 0.604 & 0.611 & 0.596 & 0.608 & 0.636 & 0.599 \\
\hline
\end{tabular}

This table reports the detrended cross-correlation coefficient ( $\rho$ (DCCA)) between investors' market participation willingness (YSI) and stock market in function of time scale $s$. (A) reports $\rho$ (DCCA) between the YSI and stock returns; (B) reports $\rho$ (DCCA) between the YSI and stock trading volume; (C) reports $\rho$ (DCCA) between the YSI and stock price volatility.

decreasing with $q$ varying from -10 to 10 . These results suggest that the cross-correlation of these two pairs is multifractal. Similar conclusions are also found in Figures 9 and 10, which shows the cross-correlation generalized Hurst exponents between market participation willingness and Chinese stock market volume and returns volatility, respectively.
Tables 4-6 report the value of cross-correlation Hurst exponents with $q$ varying from -10 to 10 and the degree of multifractality $\Delta H_{q}$. As shown in Table 4 , when $q=2$, the Hurst exponents of the cross-correlation between market participation willingness and four major stock market indices' returns, including SSE50, CSI300, SME, and GEM, are $0.486,0.552,0.553$, and 0.457 . The Hurst exponents of SSE50 


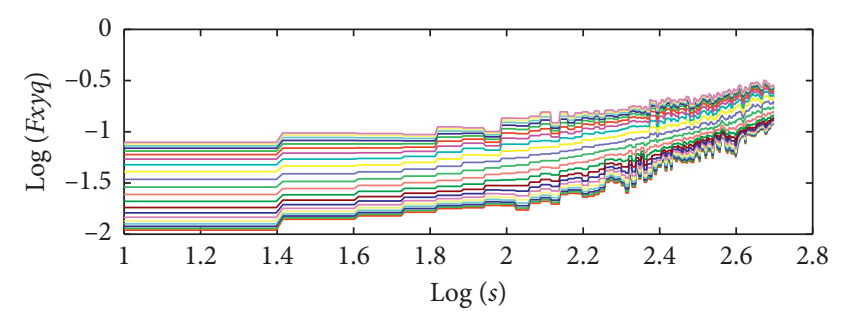

(a)

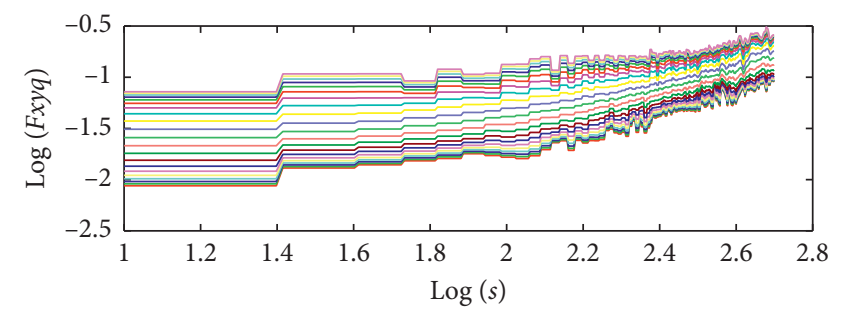

(c)

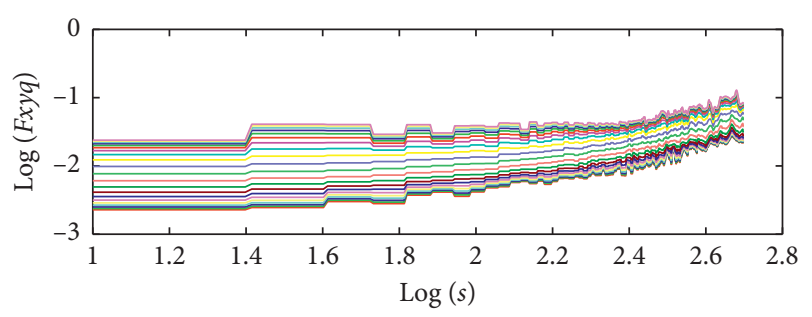

(b)

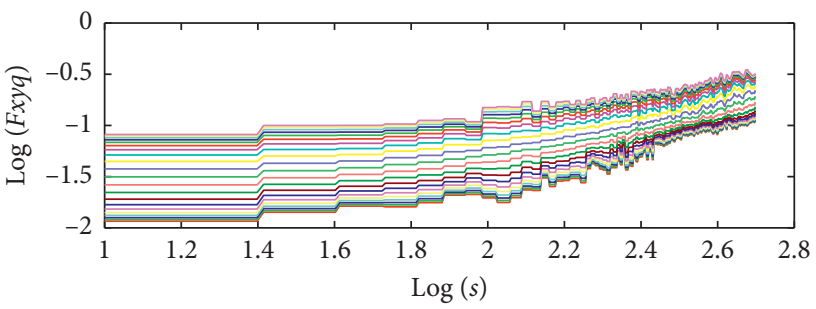

(d)

FigURE 5: Log-log plots of cross-correlation fluctuation function versus time scale $s$ for market participation willingness and Chinese stock market returns. (a) SME. (b) SSE50. (c) CSI300. (d) GEM.

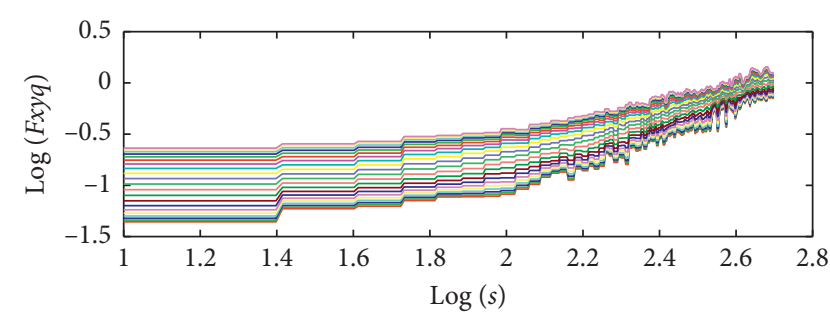

(a)

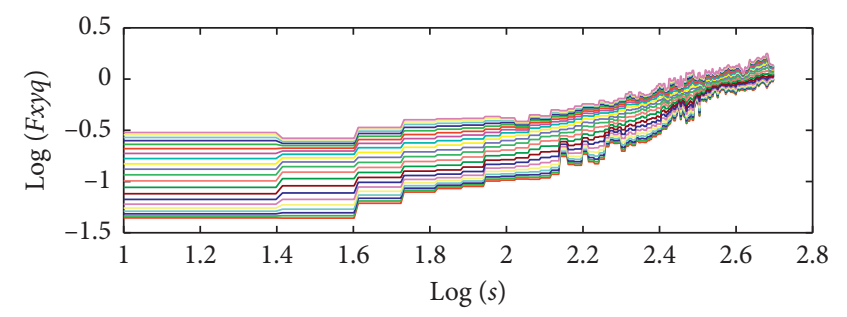

(c)

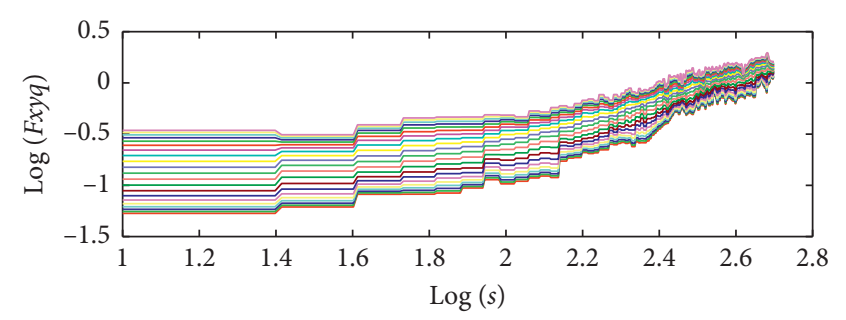

(b)

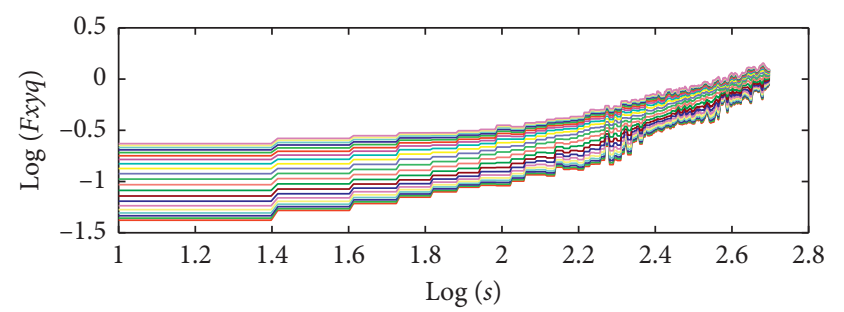

(d)

FIGURE 6: Log-log plots of cross-correlation fluctuation function versus time scale $s$ for market participation willingness and Chinese stock market volume. (a) SME. (b) SSE50. (c) CSI300. (d) GEM.

and GEM are less than 0.5 , implying that the cross-correlations between market participation willingness and SSE50 and GEM, respectively, are antipersistent; however, the Hurst exponents of CSI300 and SME are larger than 0.5 , implying that the cross-correlations between CSI300 and SME, respectively, are persistent. However, when $q=2$, all Hurst exponents of the cross-correlations between market participation willingness and Chinese stock market volume are larger than 0.5 , indicating that the cross-correlations between market participation willingness and Chinese stock market volume are strongly persistent. For returns volatility, Table 7 reports that all degrees of multifractality for the small fluctuations $(q<0)$ are smaller than the large fluctuations $(q>0)$, suggesting that the cross-correlation between market participation willingness and Chinese stock market returns volatility is more stable when the fluctuation is large. Moreover, all the Hurst exponents of cross-correlation between Chinese stock market returns volatility and market participation willingness are larger than 0.5 when $q=2$, indicating that the cross-correlations between Chinese stock market returns volatility and market participation willingness are significantly persistent.

Following Podobnik et al. [50], we can find turning points $S^{*}$ in Figures $4-6$ which indicate the fundamental change of the linear trend of the curves. There are two different behavior patterns in financial market: one is the short-term behavior which is easily influenced by the market 


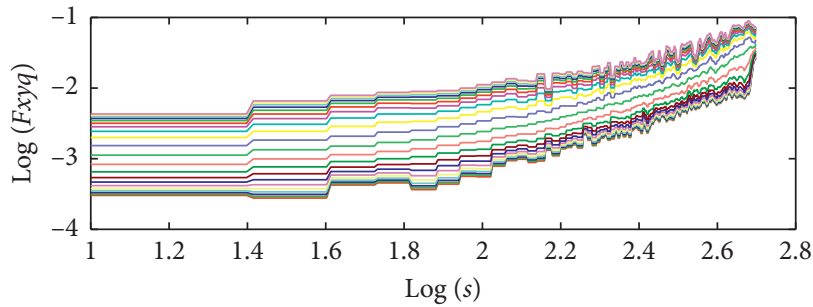

(a)

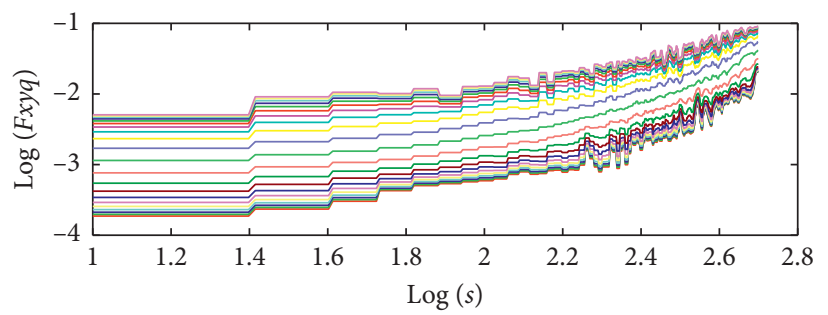

(c)

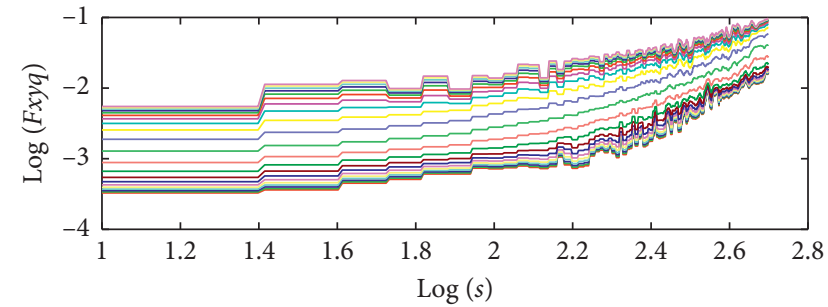

(b)

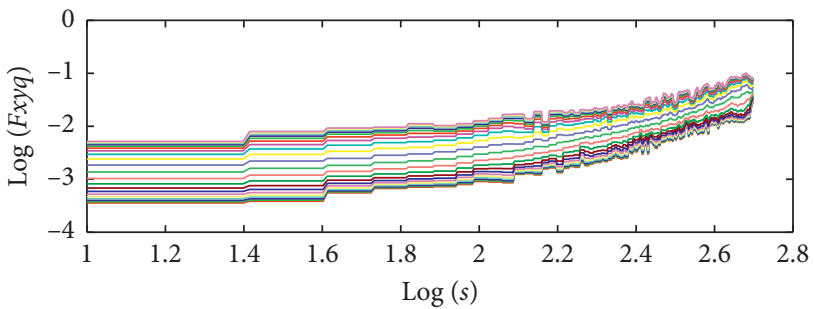

(d)

FIGURE 7: Log-log plots of cross-correlation fluctuation function versus time scale $s$ for market participation willingness and Chinese stock market returns volatility. (a) SME. (b) SSE50. (c) CSI300. (d) GEM.

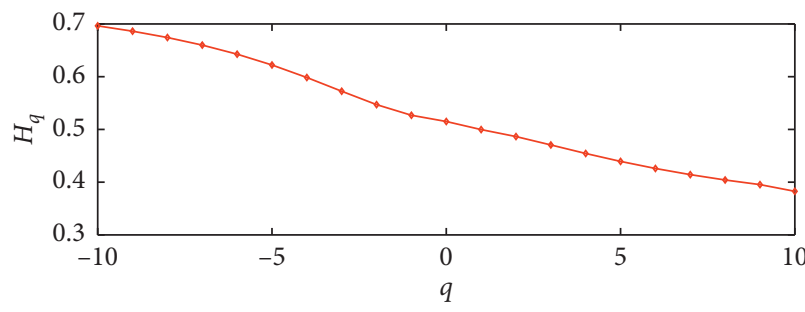

(a)

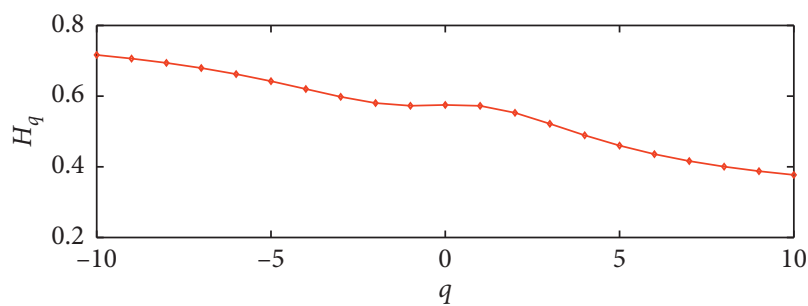

(c)

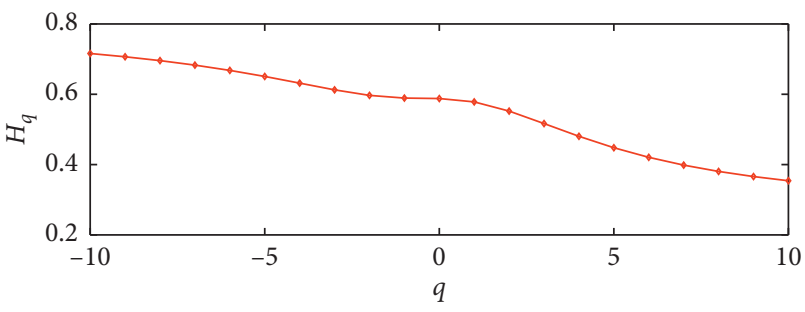

(b)

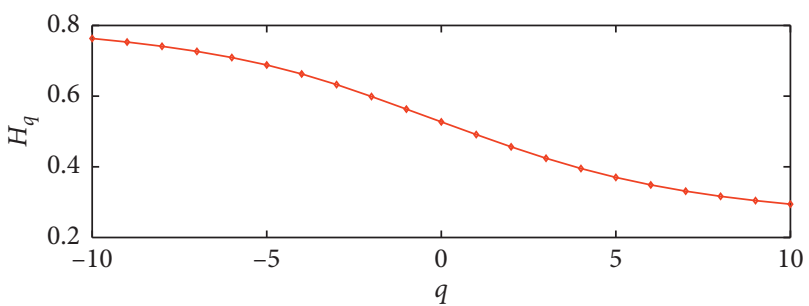

(d)

FIGURE 8: MF-DCCA scaling exponent for the market participation willingness and Chinese stock market returns. (a) SME-YSI. (b) SSE50YSI. (c) CSI300-YSI. (d) GEM-YSI.

external factors and the other is the long-term behavior which is determined by the internal factors. The scaling exponents for $s<S^{*}$ can reflect the short-range correlation and imply the short-term behaviors, while the scaling exponents for $s>S^{*}$ imply the related behaviors in the long term. As observed from Figures 4-6, we can find the turning points at about $\log 10\left(S^{*}\right)=2.3$ (about 200 days) for Chinese stock market returns, trading volume, and returns volatility. We provide the scaling exponents $H_{q}$ with $q$ varying from -10 to 10 in the short term $\left(s<S^{*}\right)$ and long term $\left(s>S^{*}\right)$ for Chinese stock market returns in Figure 11, trading volume in Figure 12, and returns volatility in
Figure 13, respectively. Tables 7-9 report the values of the scaling exponents $H_{q}$ with $q$. As shown in Table 7, the scaling exponents for $q=2$ in the long term are all smaller than 0.5 , indicating cross-correlated behaviors of large fluctuations are antipersistent in the long term for stock market return. In Table 8, we can find that all scaling exponents for $q=2$ in the short term are larger than 0.5 , implying the cross-correlated behaviors of large fluctuations are persistent in the short term for stock market volume, while the cross-correlated behaviors of large fluctuations are antipersistent in the long term for stock market volume. Table 9 reports that all the scaling exponents for $q=2$ are larger than 0.5 both in 


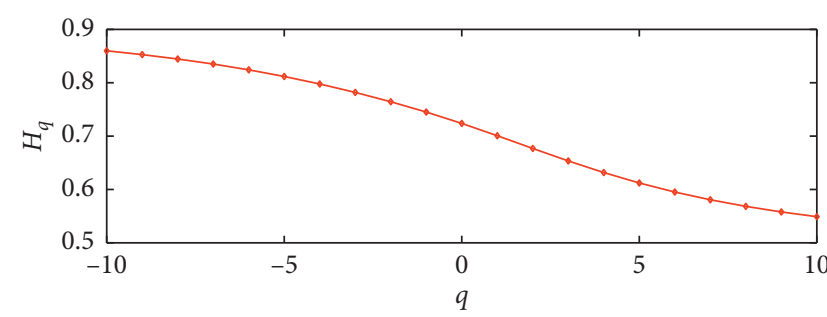

(a)

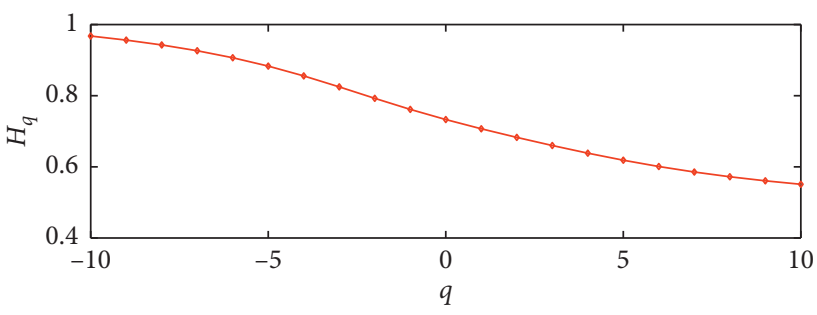

(c)

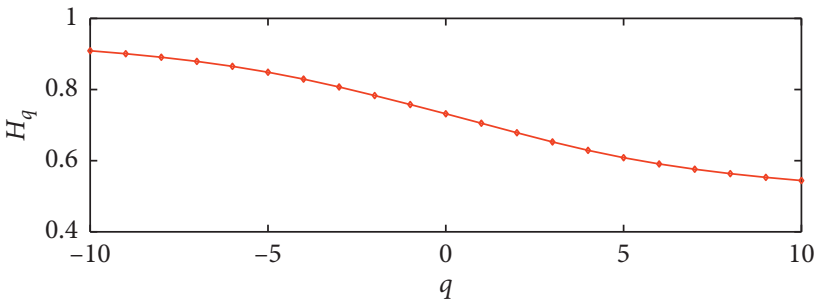

(b)

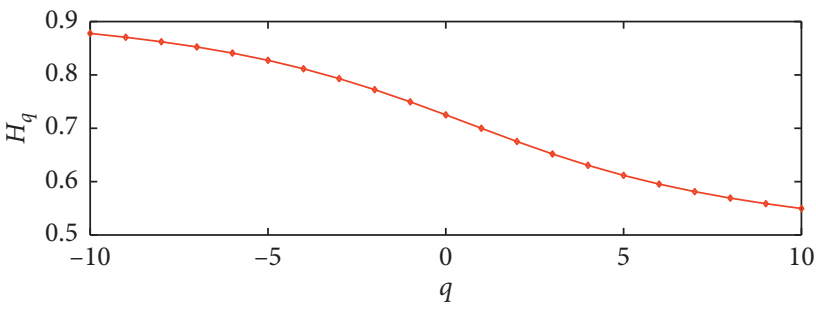

(d)

FIGURE 9: MF-DCCA scaling exponent for the market participation willingness and Chinese stock market volume. (a) SME-YSI. (b) SSE50YSI. (c) CSI300-YSI. (d) GEM-YSI.

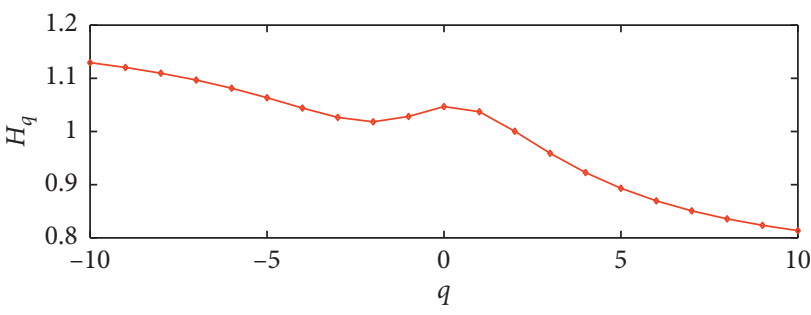

(a)

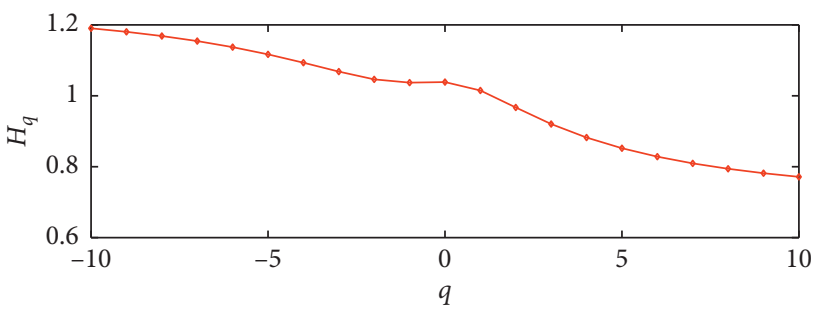

(c)

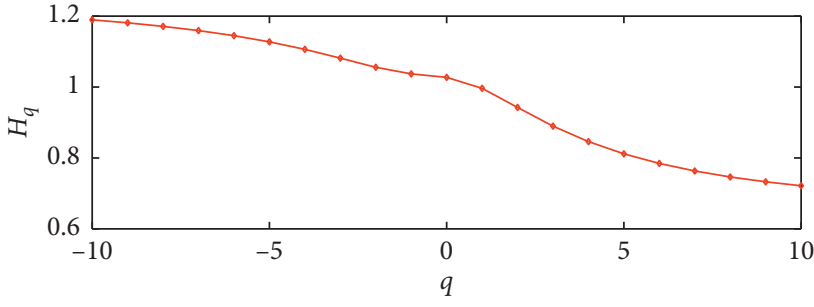

(b)

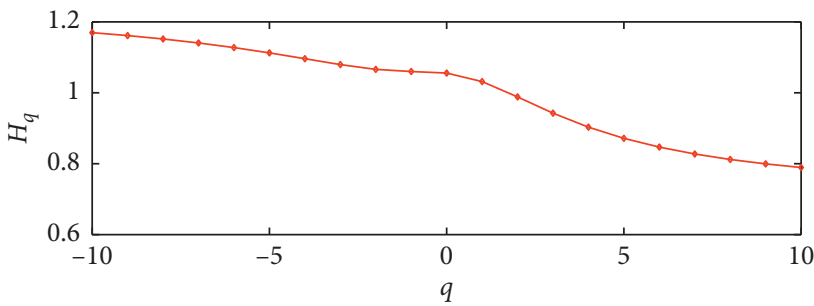

(d)

FIGURE 10: MF-DCCA scaling exponent for the market participation willingness and Chinese stock market returns volatility. (a) SME-YSI. (b) SSE50-YSI. (c) CSI300-YSI. (d) GEM-YSI.

the short term and long term, suggesting that the cross-correlated behaviors of lager fluctuations are persistent both in the short term and long term for Chinese stock market returns volatility. In addition, we find that the degrees of multifractality of the cross-correlation in the short term are smaller than those in the long term in Table 7. We can conclude that the crosscorrelations between market participation willingness and Chinese stock market returns are more stable in the short term. Furthermore, the degrees of multifractality of the cross-correlation in the short term are larger than those in the long term in Tables 8 and 9, implying that the cross-correlations between market participation willingness and Chinese stock market trading volume and returns volatility, respectively, are more stable in the long term.
4.5. Rolling Window Analysis of Cross-Correlation. It is always a hot issue in the financial field to test or model structural breaks or regime switches in financial time series [20]. A number of these models, such as Markov switching ARCH [51] and Markov switching GARCH [52], are used to capture and depict regime shifts of low-frequency volatility dynamics. Later, the rolling window analysis, which is the approach of running a sequence of estimations over a moving window of data to analyze the evolving nature of time series, has been broadly adopted to explore dynamic features of scaling exponents and examine the impacts of external shocks and forecast trends.

Following Wang et al. [53], we set the window size to 250 trading days in this paper. Figure 14 illustrates the results of 
TABLE 4: Results of the MF-DCCA scaling exponent for Chinese stock market returns.

\begin{tabular}{|c|c|c|c|c|}
\hline$q$ & SME-YSI & SSE50-YSI & CSI300-YSI & GME-YSI \\
\hline-10 & 0.696 & 0.716 & 0.717 & 0.763 \\
\hline-9 & 0.686 & 0.707 & 0.706 & 0.753 \\
\hline-8 & 0.674 & 0.696 & 0.694 & 0.741 \\
\hline-7 & 0.659 & 0.683 & 0.679 & 0.727 \\
\hline-6 & 0.643 & 0.668 & 0.662 & 0.709 \\
\hline-5 & 0.622 & 0.651 & 0.642 & 0.688 \\
\hline-4 & 0.598 & 0.632 & 0.620 & 0.663 \\
\hline-3 & 0.572 & 0.613 & 0.598 & 0.633 \\
\hline-2 & 0.547 & 0.597 & 0.580 & 0.599 \\
\hline-1 & 0.527 & 0.589 & 0.573 & 0.563 \\
\hline 0 & 0.515 & 0.587 & 0.575 & 0.527 \\
\hline$\Delta H_{q}$ & 0.181 & 0.129 & 0.142 & 0.236 \\
\hline 1 & 0.499 & 0.578 & 0.573 & 0.491 \\
\hline 2 & 0.486 & 0.552 & 0.553 & 0.457 \\
\hline 3 & 0.471 & 0.517 & 0.522 & 0.424 \\
\hline 4 & 0.454 & 0.480 & 0.489 & 0.395 \\
\hline 5 & 0.439 & 0.448 & 0.460 & 0.370 \\
\hline 6 & 0.426 & 0.421 & 0.436 & 0.349 \\
\hline 7 & 0.414 & 0.398 & 0.416 & 0.331 \\
\hline 8 & 0.404 & 0.381 & 0.400 & 0.316 \\
\hline 9 & 0.395 & 0.366 & 0.388 & 0.304 \\
\hline 10 & 0.383 & 0.354 & 0.377 & 0.294 \\
\hline$\Delta H_{q}$ & 0.116 & 0.224 & 0.196 & 0.197 \\
\hline
\end{tabular}

This table reports the MF-DCCA scaling exponent between the returns of four stock market indices and market participation willingness with $q$ varying from -10 to 10 . The symbol $\Delta H_{q}$ denotes the degree of multifractality for the large and small fluctuations of the returns and YSI.

TABLE 5: Results of the MF-DCCA scaling exponent for Chinese stock market volume.

\begin{tabular}{|c|c|c|c|c|}
\hline$q$ & SME-YSI & SSE50-YSI & CSI300-YSI & GME-YSI \\
\hline-10 & 0.859 & 0.909 & 0.968 & 0.878 \\
\hline-9 & 0.853 & 0.901 & 0.956 & 0.871 \\
\hline-8 & 0.845 & 0.891 & 0.943 & 0.862 \\
\hline-7 & 0.835 & 0.879 & 0.926 & 0.852 \\
\hline-6 & 0.824 & 0.865 & 0.907 & 0.841 \\
\hline-5 & 0.812 & 0.849 & 0.883 & 0.827 \\
\hline-4 & 0.798 & 0.829 & 0.856 & 0.812 \\
\hline-3 & 0.782 & 0.807 & 0.825 & 0.793 \\
\hline-2 & 0.764 & 0.783 & 0.792 & 0.772 \\
\hline-1 & 0.745 & 0.758 & 0.762 & 0.749 \\
\hline 0 & 0.724 & 0.732 & 0.733 & 0.725 \\
\hline$\Delta H_{q}$ & 0.135 & 0.177 & 0.235 & 0.153 \\
\hline $1^{4}$ & 0.701 & 0.705 & 0.707 & 0.700 \\
\hline 2 & 0.677 & 0.678 & 0.683 & 0.675 \\
\hline 3 & 0.653 & 0.653 & 0.659 & 0.652 \\
\hline 4 & 0.632 & 0.629 & 0.638 & 0.631 \\
\hline 5 & 0.612 & 0.608 & 0.619 & 0.612 \\
\hline 6 & 0.595 & 0.591 & 0.601 & 0.595 \\
\hline 7 & 0.581 & 0.576 & 0.585 & 0.581 \\
\hline 8 & 0.568 & 0.563 & 0.572 & 0.569 \\
\hline 9 & 0.558 & 0.553 & 0.561 & 0.559 \\
\hline 10 & 0.549 & 0.544 & 0.551 & 0.549 \\
\hline$\Delta H_{q}$ & 0.152 & 0.161 & 0.156 & 0.151 \\
\hline
\end{tabular}

This table reports the MF-DCCA scaling exponent between the volume of four stock market indices and market participation willingness with $q$ varying from -10 to 10 . The symbol $\Delta H_{q}$ denotes the degree of multifractality for the large and small fluctuations of the returns and YSI.

the rolling window analysis for the cross-correlation between market participation willingness and Chinese stock market return. As observed from Figure 14, the cross- correlation exponents fluctuated violently around the level of 0.5 with exponents smaller than 0.5 accounting for the vast majority. These results imply the fact that mostly, the 
TABLE 6: Results of the MF-DCCA scaling exponent for Chinese stock market returns volatility.

\begin{tabular}{|c|c|c|c|c|}
\hline$q$ & SME-YSI & SSE50-YSI & CSI300-YSI & GME-YSI \\
\hline-10 & 1.129 & 1.189 & 1.190 & 1.169 \\
\hline-9 & 1.120 & 1.181 & 1.181 & 1.161 \\
\hline-8 & 1.109 & 1.171 & 1.169 & 1.152 \\
\hline-7 & 1.097 & 1.159 & 1.154 & 1.141 \\
\hline-6 & 1.081 & 1.145 & 1.137 & 1.128 \\
\hline-5 & 1.064 & 1.127 & 1.117 & 1.113 \\
\hline-4 & 1.044 & 1.106 & 1.093 & 1.096 \\
\hline-3 & 1.026 & 1.082 & 1.068 & 1.079 \\
\hline-2 & 1.018 & 1.056 & 1.046 & 1.066 \\
\hline-1 & 1.028 & 1.037 & 1.037 & 1.060 \\
\hline 0 & 1.047 & 1.027 & 1.039 & 1.056 \\
\hline$\Delta H_{q}$ & 0.082 & 0.162 & 0.161 & 0.113 \\
\hline $1^{4}$ & 1.037 & 0.996 & 1.015 & 1.032 \\
\hline 2 & 1.001 & 0.942 & 0.967 & 0.988 \\
\hline 3 & 0.959 & 0.889 & 0.920 & 0.943 \\
\hline 4 & 0.923 & 0.846 & 0.882 & 0.903 \\
\hline 5 & 0.893 & 0.812 & 0.852 & 0.872 \\
\hline 6 & 0.869 & 0.785 & 0.828 & 0.847 \\
\hline 7 & 0.851 & 0.763 & 0.809 & 0.827 \\
\hline 8 & 0.836 & 0.746 & 0.794 & 0.812 \\
\hline 9 & 0.824 & 0.733 & 0.782 & 0.799 \\
\hline 10 & 0.814 & 0.722 & 0.771 & 0.789 \\
\hline$\Delta H_{q}$ & 0.223 & 0.274 & 0.244 & 0.243 \\
\hline
\end{tabular}

This table reports the MF-DCCA scaling exponent between the volatility of four stock market indices and market participation willingness with $q$ varying from -10 to 10 . The symbol $\Delta H_{q}$ denotes the degree of multifractality for the large and small fluctuations of the returns and YSI.

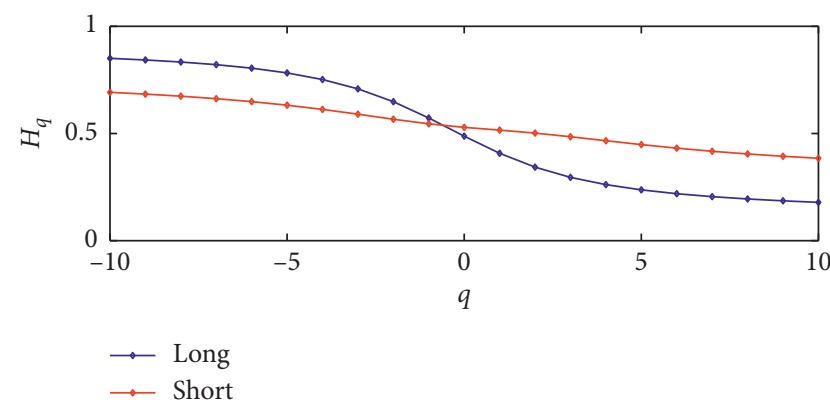

(a)

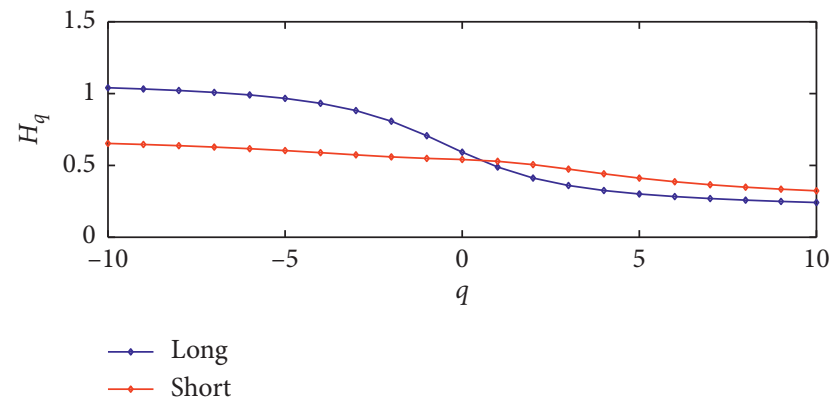

(c)

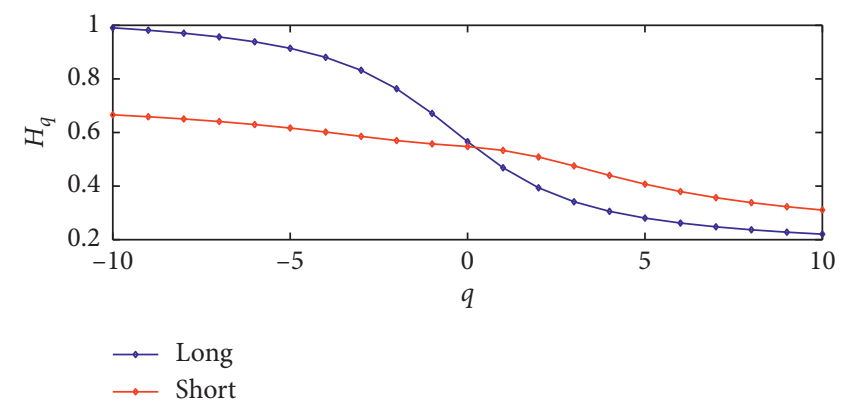

(b)

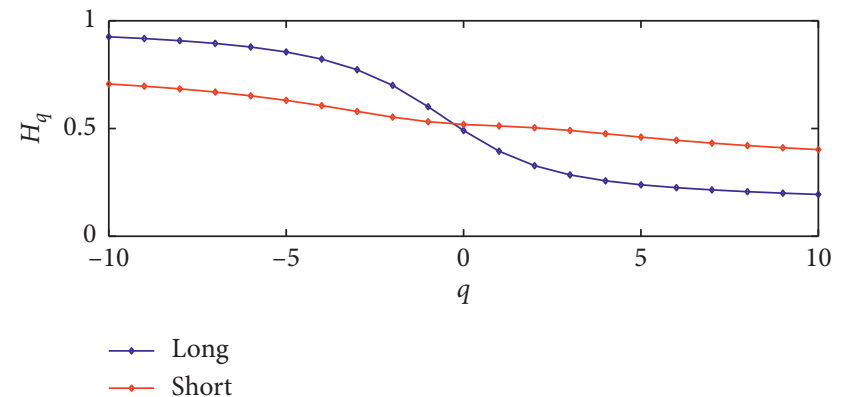

(d)

FIGURE 11: Short and long scaling exponents for Chinese stock market returns and market participation willingness. (a) SME-YSI. (b) SSE50-YSI. (c) CSI300-YSI. (d) GEM-YSI. 

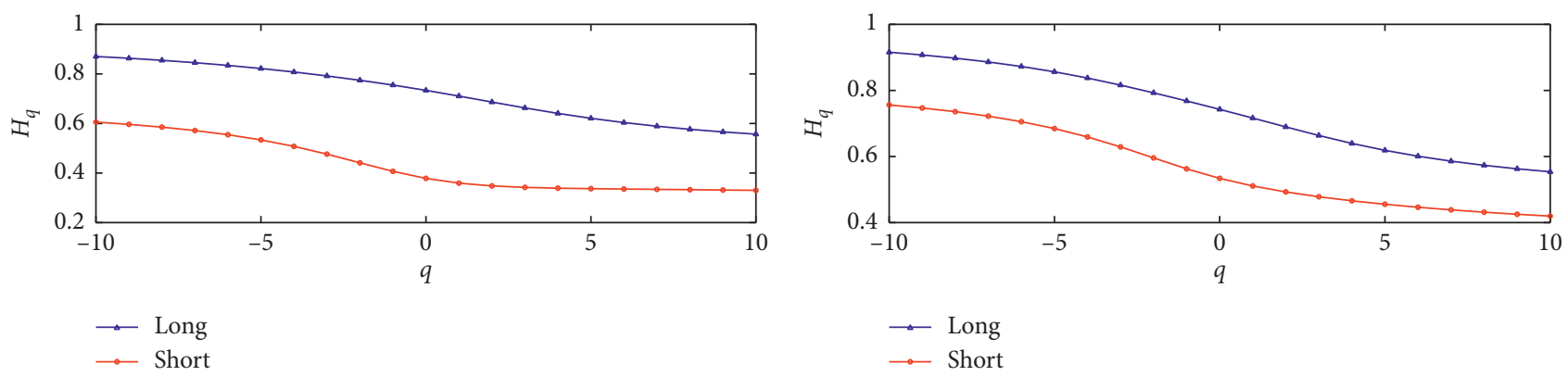

(a)
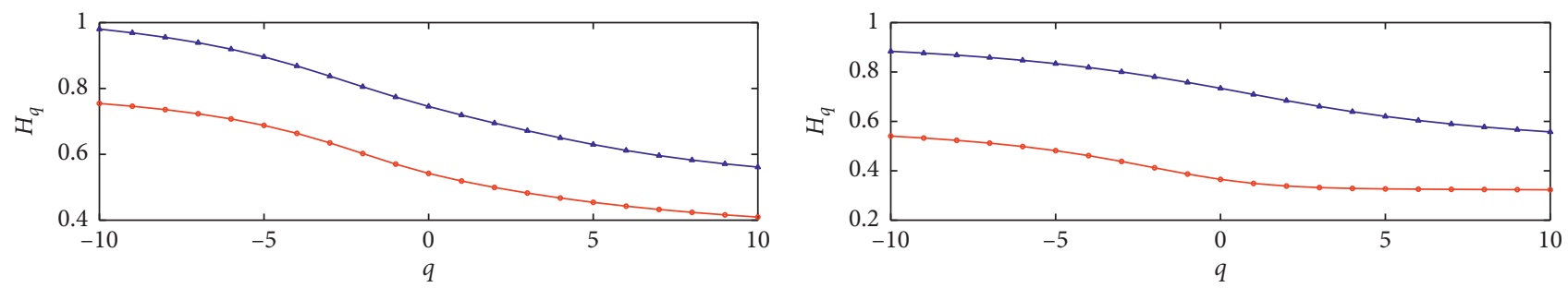

$\rightarrow$ Long
$\rightarrow$ Short

$\rightarrow$ Long
$\rightarrow$ Short

(c)

(d)

FIGURE 12: Short and long scaling exponents for Chinese stock market volume and market participation willingness. (a) SME-YSI. (b) SSE50-YSI. (c) CSI300-YSI. (d) GEM-YSI.
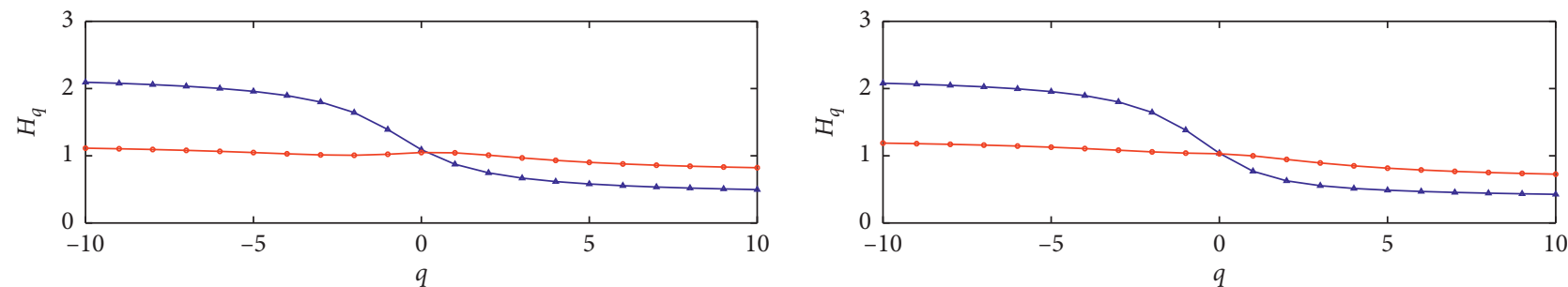

$\rightarrow$ Long

$\rightarrow$ Long

$\rightarrow$ Short

$\rightarrow$ Short

(a)
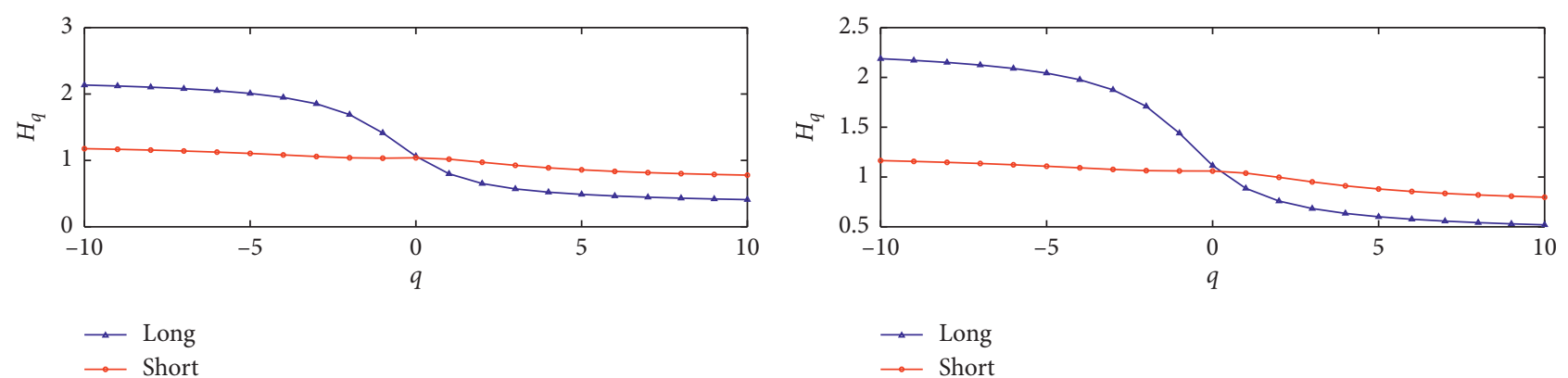

(c)

(d)

FIGURE 13: Short and long scaling exponents for Chinese stock market volatility and market participation willingness. (a) SME-YSI. (b) SSE50-YSI. (c) CSI300-YSI. (d) GEM-YSI.

cross-correlations between market participation willingness and Chinese market returns exhibit antipersistent features. Figure 15 illustrates the results of the rolling window analysis for the cross-correlation between market participation willingness and Chinese stock market trading volume. And we can observe that the cross-correlation exponents 
TABLE 7: Results of the short-term and long-term scaling exponents for Chinese stock market returns.

\begin{tabular}{|c|c|c|c|c|c|c|c|c|}
\hline \multirow{2}{*}{$q$} & \multicolumn{2}{|c|}{ SME-YSI } & \multicolumn{2}{|c|}{ SSE50-YSI } & \multicolumn{2}{|c|}{ CSI300-YSI } & \multicolumn{2}{|c|}{ GME-YSI } \\
\hline & Short & Long & Short & Long & Short & Long & Short & Long \\
\hline-10 & 0.692 & 0.851 & 0.666 & 0.991 & 0.653 & 1.041 & 0.707 & 0.926 \\
\hline-9 & 0.684 & 0.843 & 0.659 & 0.982 & 0.646 & 1.032 & 0.696 & 0.918 \\
\hline-8 & 0.674 & 0.834 & 0.651 & 0.971 & 0.637 & 1.022 & 0.684 & 0.908 \\
\hline-7 & 0.662 & 0.821 & 0.641 & 0.957 & 0.628 & 1.008 & 0.669 & 0.895 \\
\hline-6 & 0.649 & 0.805 & 0.629 & 0.938 & 0.616 & 0.991 & 0.652 & 0.878 \\
\hline-5 & 0.632 & 0.783 & 0.617 & 0.914 & 0.603 & 0.967 & 0.631 & 0.855 \\
\hline-4 & 0.612 & 0.752 & 0.602 & 0.881 & 0.589 & 0.932 & 0.606 & 0.822 \\
\hline-3 & 0.590 & 0.708 & 0.585 & 0.832 & 0.573 & 0.882 & 0.579 & 0.773 \\
\hline-2 & 0.566 & 0.648 & 0.569 & 0.763 & 0.559 & 0.808 & 0.553 & 0.700 \\
\hline-1 & 0.545 & 0.572 & 0.557 & 0.671 & 0.549 & 0.707 & 0.531 & 0.601 \\
\hline 0 & 0.528 & 0.487 & 0.547 & 0.565 & 0.541 & 0.592 & 0.519 & 0.491 \\
\hline 1 & 0.516 & 0.408 & 0.533 & 0.468 & 0.529 & 0.4889 & 0.512 & 0.395 \\
\hline 2 & 0.502 & 0.343 & 0.509 & 0.394 & 0.506 & 0.412 & 0.504 & 0.327 \\
\hline 3 & 0.485 & 0.295 & 0.475 & 0.341 & 0.474 & 0.360 & 0.491 & 0.285 \\
\hline 4 & 0.466 & 0.261 & 0.439 & 0.306 & 0.442 & 0.325 & 0.476 & 0.257 \\
\hline 5 & 0.448 & 0.237 & 0.407 & 0.281 & 0.412 & 0.301 & 0.460 & 0.239 \\
\hline 6 & 0.432 & 0.219 & 0.379 & 0.262 & 0.386 & 0.283 & 0.445 & 0.225 \\
\hline 7 & 0.417 & 0.205 & 0.357 & 0.248 & 0.366 & 0.269 & 0.432 & 0.215 \\
\hline 8 & 0.404 & 0.194 & 0.338 & 0.237 & 0.349 & 0.258 & 0.421 & 0.207 \\
\hline 9 & 0.394 & 0.186 & 0.323 & 0.228 & 0.335 & 0.249 & 0.411 & 0.199 \\
\hline 10 & 0.384 & 0.179 & 0.311 & 0.221 & 0.323 & 0.242 & 0.402 & 0.194 \\
\hline$\Delta H_{q}$ & 0.308 & 0.672 & 0.355 & 0.770 & 0.330 & 0.799 & 0.305 & 0.732 \\
\hline
\end{tabular}

This table reports the short-term and long-term scaling exponents between the returns of four stock market indices and market participation willingness with $q$ varying from -10 to $10 . \Delta H_{q}$ is the multifractality degree. "Short" denotes $s<S^{*}$ and "Long" denotes $s>S^{*}$.

TABLE 8: Results of the short-term and long-term scaling exponents for Chinese stock market volume.

\begin{tabular}{|c|c|c|c|c|c|c|c|c|}
\hline \multirow[b]{2}{*}{$q$} & \multicolumn{2}{|c|}{ SME-YSI } & \multicolumn{2}{|c|}{ SSE50-YSI } & \multicolumn{2}{|c|}{ CSI300-YSI } & \multicolumn{2}{|c|}{ GME-YSI } \\
\hline & Short & Long & Short & Long & Short & Long & Short & Long \\
\hline-10 & 0.870 & 0.606 & 0.916 & 0.756 & 0.980 & 0.755 & 0.884 & 0.541 \\
\hline-9 & 0.863 & 0.596 & 0.907 & 0.747 & 0.969 & 0.746 & 0.876 & 0.533 \\
\hline-8 & 0.855 & 0.585 & 0.898 & 0.736 & 0.955 & 0.736 & 0.868 & 0.523 \\
\hline-7 & 0.845 & 0.572 & 0.886 & 0.722 & 0.939 & 0.723 & 0.859 & 0.512 \\
\hline-6 & 0.834 & 0.555 & 0.872 & 0.705 & 0.919 & 0.707 & 0.847 & 0.498 \\
\hline-5 & 0.822 & 0.534 & 0.856 & 0.685 & 0.894 & 0.688 & 0.834 & 0.482 \\
\hline-4 & 0.808 & 0.507 & 0.838 & 0.659 & 0.868 & 0.664 & 0.818 & 0.461 \\
\hline-3 & 0.792 & 0.476 & 0.816 & 0.629 & 0.837 & 0.635 & 0.800 & 0.438 \\
\hline-2 & 0.774 & 0.441 & 0.793 & 0.596 & 0.805 & 0.602 & 0.780 & 0.412 \\
\hline-1 & 0.755 & 0.407 & 0.768 & 0.563 & 0.774 & 0.570 & 0.758 & 0.387 \\
\hline 0 & 0.734 & 0.378 & 0.743 & 0.534 & 0.745 & 0.542 & 0.734 & 0.365 \\
\hline 1 & 0.711 & 0.359 & 0.716 & 0.511 & 0.719 & 0.519 & 0.709 & 0.349 \\
\hline 2 & 0.686 & 0.348 & 0.689 & 0.493 & 0.695 & 0.499 & 0.684 & 0.338 \\
\hline 3 & 0.663 & 0.342 & 0.664 & 0.478 & 0.672 & 0.482 & 0.661 & 0.332 \\
\hline 4 & 0.641 & 0.339 & 0.639 & 0.466 & 0.649 & 0.467 & 0.639 & 0.328 \\
\hline 5 & 0.621 & 0.337 & 0.619 & 0.455 & 0.629 & 0.454 & 0.620 & 0.327 \\
\hline 6 & 0.603 & 0.335 & 0.601 & 0.446 & 0.612 & 0.443 & 0.604 & 0.326 \\
\hline 7 & 0.589 & 0.334 & 0.586 & 0.438 & 0.596 & 0.433 & 0.589 & 0.325 \\
\hline 8 & 0.576 & 0.333 & 0.573 & 0.431 & 0.583 & 0.424 & 0.577 & 0.324 \\
\hline 9 & 0.566 & 0.331 & 0.563 & 0.425 & 0.571 & 0.416 & 0.567 & 0.324 \\
\hline 10 & 0.557 & 0.330 & 0.554 & 0.419 & 0.561 & 0.409 & 0.557 & 0.323 \\
\hline$\Delta H_{q}$ & 0.313 & 0.276 & 0.362 & 0.337 & 0.419 & 0.346 & 0.327 & 0.218 \\
\hline
\end{tabular}

This table reports the short-term and long-term scaling exponents between the volume of four stock market indices and market participation willingness with $q$ varying from -10 to $10 . \Delta H_{q}$ is the multifractality degree. "Short" denotes $s<S^{*}$ and "Long" the $s>S^{*}$.

fluctuated violently all beyond the level of 0.5 , indicating that the cross-correlations between market participation willingness and Chinese market volume exhibit persistent features in the whole period. Similar conclusions are also found in Figure 16, which illustrates the cross-correlation between market participation willingness and Chinese stock 
TABLE 9: Results of the short-term and long-term scaling exponents for Chinese stock market returns volatility.

\begin{tabular}{|c|c|c|c|c|c|c|c|c|}
\hline \multirow{2}{*}{$q$} & \multicolumn{2}{|c|}{ SME-YSI } & \multicolumn{2}{|c|}{ SSE50-YSI } & \multicolumn{2}{|c|}{ CSI300-YSI } & \multicolumn{2}{|c|}{ GME-YSI } \\
\hline & Short & Long & Short & Long & Short & Long & Short & Long \\
\hline-10 & 2.094 & 1.113 & 2.079 & 1.188 & 2.135 & 1.177 & 2.188 & 1.165 \\
\hline-9 & 2.078 & 1.104 & 2.065 & 1.180 & 2.121 & 1.167 & 2.171 & 1.157 \\
\hline-8 & 2.059 & 1.093 & 2.048 & 1.170 & 2.103 & 1.156 & 2.151 & 1.147 \\
\hline-7 & 2.034 & 1.081 & 2.026 & 1.159 & 2.080 & 1.142 & 2.124 & 1.136 \\
\hline-6 & 2.002 & 1.065 & 1.996 & 1.145 & 2.050 & 1.125 & 2.089 & 1.123 \\
\hline-5 & 1.959 & 1.048 & 1.955 & 1.128 & 2.009 & 1.105 & 2.043 & 1.108 \\
\hline-4 & 1.896 & 1.029 & 1.895 & 1.107 & 1.948 & 1.083 & 1.977 & 1.092 \\
\hline-3 & 1.800 & 1.013 & 1.802 & 1.083 & 1.853 & 1.059 & 1.875 & 1.076 \\
\hline-2 & 1.643 & 1.007 & 1.645 & 1.058 & 1.690 & 1.039 & 1.709 & 1.064 \\
\hline-1 & 1.391 & 1.023 & 1.382 & 1.039 & 1.414 & 1.033 & 1.440 & 1.061 \\
\hline 0 & 1.090 & 1.048 & 1.038 & 1.030 & 1.063 & 1.039 & 1.115 & 1.060 \\
\hline 1 & 0.874 & 1.044 & 0.769 & 0.999 & 0.798 & 1.019 & 0.886 & 1.039 \\
\hline 2 & 0.746 & 1.009 & 0.627 & 0.945 & 0.652 & 0.972 & 0.758 & 0.997 \\
\hline 3 & 0.667 & 0.968 & 0.555 & 0.893 & 0.571 & 0.926 & 0.683 & 0.951 \\
\hline 4 & 0.616 & 0.932 & 0.514 & 0.850 & 0.522 & 0.888 & 0.635 & 0.911 \\
\hline 5 & 0.581 & 0.902 & 0.488 & 0.816 & 0.488 & 0.858 & 0.601 & 0.880 \\
\hline 6 & 0.555 & 0.878 & 0.469 & 0.789 & 0.464 & 0.835 & 0.576 & 0.855 \\
\hline 7 & 0.535 & 0.860 & 0.455 & 0.768 & 0.446 & 0.816 & 0.557 & 0.835 \\
\hline 8 & 0.519 & 0.845 & 0.444 & 0.751 & 0.431 & 0.801 & 0.542 & 0.820 \\
\hline 9 & 0.507 & 0.833 & 0.435 & 0.737 & 0.419 & 0.788 & 0.530 & 0.807 \\
\hline 10 & 0.497 & 0.823 & 0.427 & 0.726 & 0.409 & 0.778 & 0.520 & 0.797 \\
\hline$\Delta H_{q}$ & 1.597 & 0.290 & 1.652 & 0.462 & 1.726 & 0.399 & 1.668 & 0.368 \\
\hline
\end{tabular}

This table reports the short-term and long-term scaling exponents between the returns volatility of four stock market indices and market participation willingness with $q$ varying from -10 to $10 . \Delta H_{q}$ is the multifractality degree. "Short" denotes $s<S^{*}$ and "Long" denotes $s>S^{*}$.

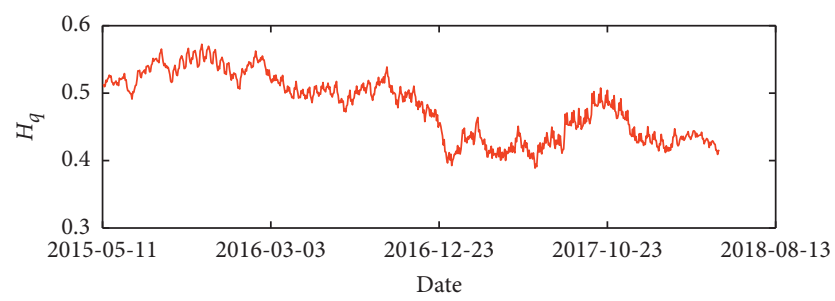

(a)

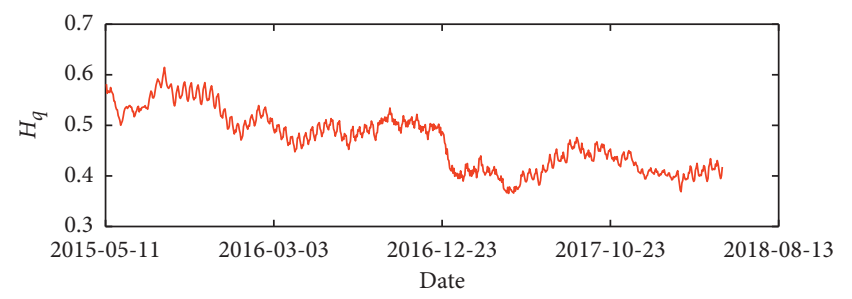

(c)

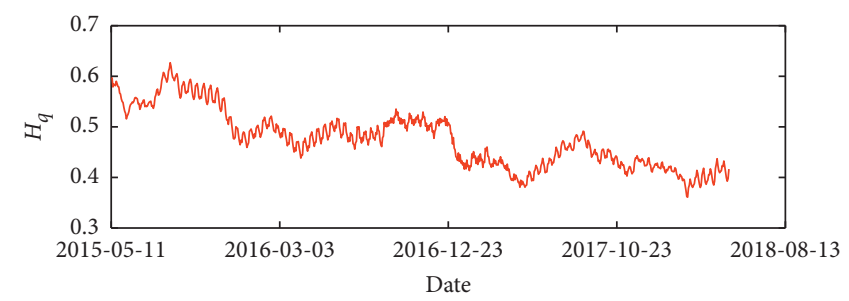

(b)

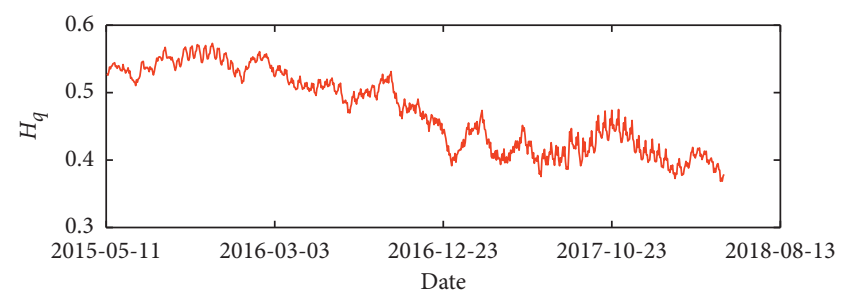

(d)

FIGURE 14: The time-varying scaling exponents for $q=2$ for Chinese stock market returns and market participation willingness when window size is 250 days. (a) SME-YSI. (b) SSE50-YSI. (c) CSI300-YSI. (d) GEM-YSI.

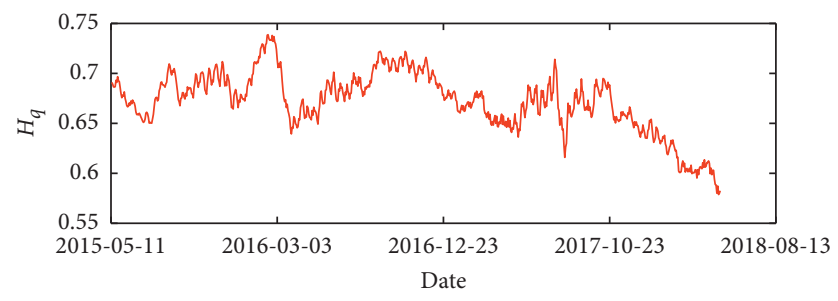

(a)

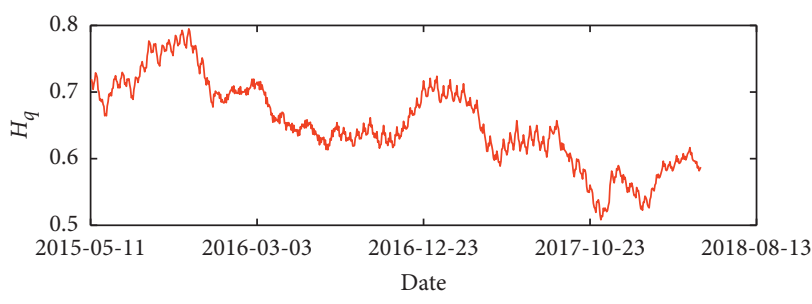

(b)

Figure 15: Continued. 


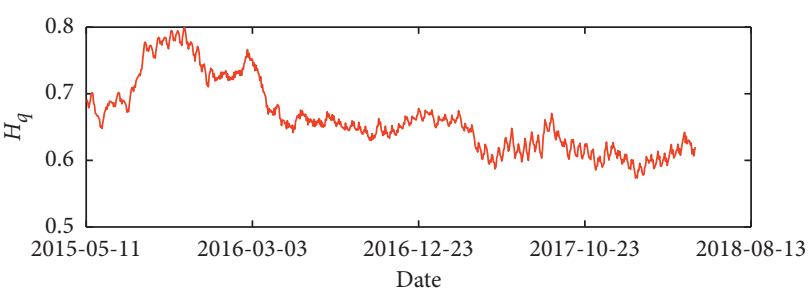

(c)

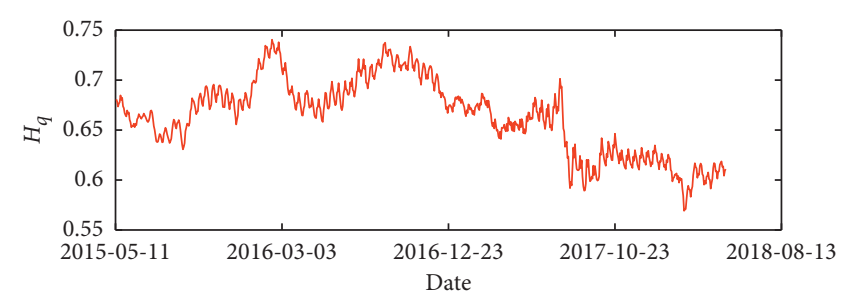

(d)

FIGURE 15: The time-varying scaling exponents for Chinese stock market volume and market participation willingness when window size is 250 days. (a) SME-YSI. (b) SSE50-YSI. (c) CSI300-YSI. (d) GEM-YSI.

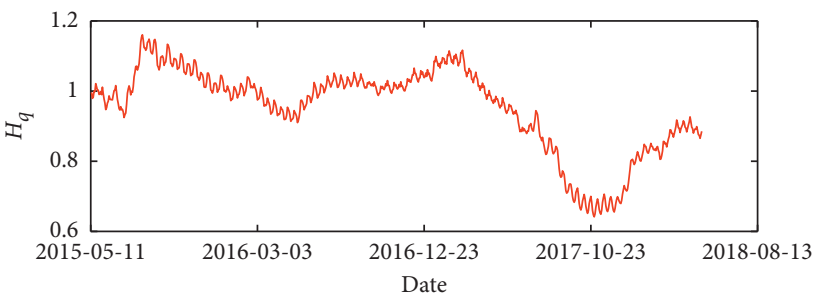

(a)

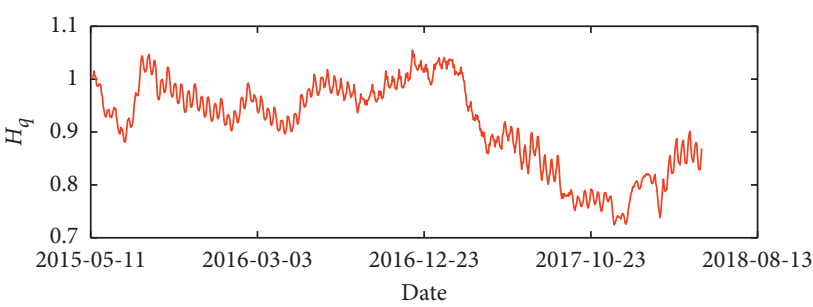

(c)

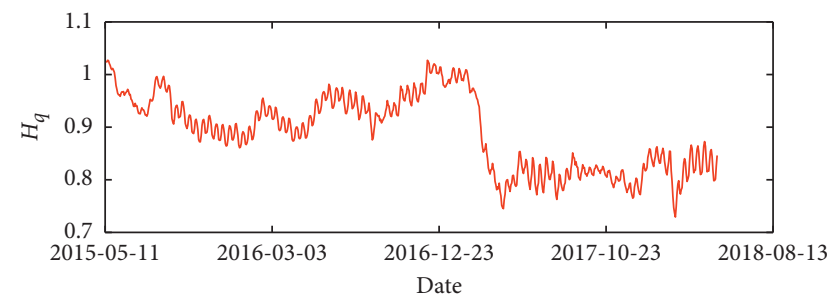

(b)

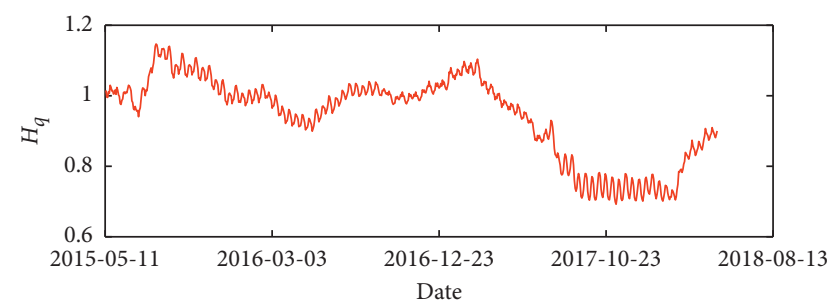

(d)

FIGURE 16: The time-varying scaling exponents for Chinese stock market volatility and market participation willingness when window size is 250 days. (a) SME-YSI. (b) SSE50-YSI. (c) CSI300-YSI. (d) GEM-YSI.

market returns volatility. The evolution of the cross-correlation exponents experienced erratic fluctuations and can be associated with financial events. For example, when Chinese stock exchanges introduced the circuit breakers in January 4, 2016, all cross-correlation exponents, both stock return and volume, were down.

\section{Conclusions}

In this paper, we investigate the nonlinear and dynamic relationship between market participation willingness, measured by Yu'e Bao Sentiment Index, and Chinese stock market performance. We utilize daily returns, trading volume, and returns volatility of SSE50, CSI300, SME, and GME to characterize the dynamic of Chinese stock market.

We employ mutual information to investigate the nonlinear dependence between the market participation willingness and Chinese stock market performance and find that there is overall dependence, both linear and nonlinear, between market participation willingness and Chinese stock market performance. Moreover, after cross-correlation test and DCCA cross-correlation coefficient analysis, we verify the existence of long-range cross-correlation between market participation willingness and stock market returns, trading volume, and volatility, respectively. We further employ MF-DCCA and find that there exists antipersistent cross-correlation between market participation willingness and Chinese stock market returns. We also find that there exists strong persistent cross-correlation between market participation willingness and Chinese stock market trading volume and returns volatility, respectively. Furthermore, we analyze the cross-correlation between market participation willingness and stock market in the short term and long term. We find that there is an antipersistent cross-correlation in the long term for market participation willingness and Chinese stock market returns and trading volume, respectively. And the cross-correlations between market participation willingness and Chinese stock returns volatility are strongly persistent both in the short term and long term. Moreover, cross-correlations between market participation willingness and Chinese stock market returns are more stable in the short term, but those between market participation willingness and Chinese stock market volume and returns volatility, respectively, are more stable in the long 
term. Using the method of rolling windows, we find that the cross-correlation exponents between market participation willingness and Chinese stock market return are close to 0.5 over time and those between market participation willingness and stock market volume and returns volatility, respectively, are larger than 0.5 . The evolution of crosscorrelation exponents experienced erratic fluctuations.

Furthermore, several investment strategies and policy implications based on the findings can be derived. For investment managers, market participation willingness is an important factor to affect the stock market performance, which should be considered in investment portfolios. Moreover, regulators should take market participation willingness as an aspect of reflecting the market operation. Investors' market participation willingness should become an important reference of making or assessing the regulatory policies. For retail investors, market participation willingness can be considered as a valuable factor to predict stock market performance and help them make reasonable investment decision.

\section{Data Availability}

The data used to support the findings of this study are available from the corresponding author upon request.

\section{Conflicts of Interest}

The authors declare that they have no conflicts of interest.

\section{Acknowledgments}

This study was supported by the National Natural Science Foundation of China (71801136, 71771170, and U1811462) and the Fundamental Research Funds for the Central Universities (63202062).

\section{References}

[1] F. Allen and D. Gale, "Limited market participation and volatility of asset prices," The American Economic Review, vol. 84, no. 4, pp. 933-955, 1994.

[2] H. Guo, "Limited stock market participation and asset prices in a dynamic economy," Journal of Financial and Quantitative Analysis, vol. 39, no. 3, pp. 495-516, 2004.

[3] A. Vissing-Jorgensen, Towards an Explanation of Household Portfolio Choice Heterogeneity: Non Financial Income and Participation Cost Structures, National Bureau of Economic Research, Cambridge, MA, USA, 2002.

[4] P. J. Bayer, B. D. Bernheim, and J. K. Scholz, "The effects of financial education in the workplace: evidence from a survey of employers financial education in the workplace: evidence from a survey of employers," Economic Inquiry, vol. 47, no. 4, pp. 605-624, 2009.

[5] M. Grinblatt, M. Keloharju, and J. Linnainmaa, "IQ and stock market participation," The Journal of Finance, vol. 66, no. 6, pp. 2121-2164, 2011.

[6] J. Almenberg and A. Dreber, "Gender, stock market participation and financial literacy," Economics Letters, vol. 137, pp. 140-142, 2015.
[7] M. Van Rooij, A. Lusardi, and R. Alessie, "Financial literacy and stock market participation," Journal of Financial Economics, vol. 101, no. 2, pp. 449-472, 2011.

[8] P. Liang and S. Guo, "Social interaction, internet access and stock market participation-an empirical study in China," Journal of Comparative Economics, vol. 43, no. 4, pp. 883-901, 2015.

[9] Y. Bonaparte and A. Kumar, "Political activism, information costs, and stock market participation," Journal of Financial Economics, vol. 107, no. 3, pp. 760-786, 2013.

[10] Y. Rao, L. Mei, and R. Zhu, "Happiness and stock-market participation: empirical evidence from China," Journal of Happiness Studies, vol. 17, no. 1, pp. 271-293, 2016.

[11] S. Basak and D. Cuoco, "An equilibrium model with restricted stock market participation," Review of Financial Studies, vol. 11, no. 2, pp. 309-341, 1998.

[12] H. Guo, "Limited stock market participation and asset prices in a dynamic economy," Journal of Financial and Quantitative Analysis, vol. 39, no. 3, pp. 495-516, 2003.

[13] V. Polkovnichenko, "Limited stock market participation and the equity premium," Finance Research Letters, vol. 1, no. 1, pp. 24-34, 2004.

[14] L. Zhang, "Local equity market participation and stock liquidity," The Quarterly Review of Economics and Finance, vol. 63, pp. 101-121, 2017.

[15] Y. D. Wang, L. Liu, and R. B. Gu, "Analysis of efficiency for Shenzhen stock market based on multifractal detrended fluctuation analysis," International Review of Financial Analysis, vol. 18, no. 5, pp. 271-276, 2009.

[16] G. X. Cao, L. B. Xu, and J. Cao, "Multifractal detrended crosscorrelations between the Chinese exchange market and stock market," Physica A: Statistical Mechanics and Its Applications, vol. 391, pp. 4855-4866, 2012.

[17] Y. H. Yang, W.-J. Xie, M.-X. Li, Z.-Q. Jiang, and W.-X. Zhou, "Statistical properties of user activity fluctuations in virtual worlds," Chaos, Solitons \& Fractals, vol. 105, pp. 271-278, 2017.

[18] C. K. Peng, S. V. Buldyrev, S. Havlin, M. Simons, and A. L. Goldberger, "Mosaic organization of DNA nucleotides," Physical Review E Statistical Physics Plasmas Fluids \& Related Interdisciplinary Topics, vol. 49, no. 2, pp. 1685-1689, 1994.

[19] S. P. Li, X. S. Lu, and X. H. Liu, "Dynamic relationship between Chinese RMB exchange rate index and market anxiety: a new perspective based on MF-DCCA," Physica A: Statistical Mechanics and Its Applications, vol. 541, Article ID 123405, 2020.

[20] X. S. Lu, X. X. Sun, and J. T. Ge, "Dynamic relationship between Japanese Yen exchange rates and market anxiety: a new perspective based on MF-DCCA," Physica A: Statistical Mechanics and Its Applications, vol. 474, pp. 144-161, 2017.

[21] G. X. Cao, Y. Han, Q. C. Li, and W. Xu, "Asymmetric MFDCCA method based on risk conduction and its application in the Chinese and foreign stock markets," Physica A: Statistical Mechanics and Its Applications, vol. 468, pp. 119-130, 2017.

[22] Q. S. Ruan, H. Q. Yang, D. Y. Lv, and S. H. Zhang, "Crosscorrelation between individual investor sentiment and Chinese stock market return: new perspective based on MFDCCA," Physica A: Statistical Mechanics and Its Applications, vol. 503, pp. 243-256, 2018.

[23] M. Bonato, K. Gkillas, R. Gupta, and C. Pierdzioch, "Investor happiness and predictability of the realized volatility of oil price," Sustainability, vol. 12, no. 10, p. 4309, 2020. 
[24] M. Bonato, K. Gkillas, R. Gupta, and C. Pierdzioch, "A note on investor happiness and the predictability of realized volatility of gold," Finance Research Letters, 2020.

[25] L. Bijl, G. Kringhaug, P. Molnár, and E. Sandvik, "Google searches and stock returns," International Review of Financial Analysis, vol. 45, pp. 150-156, 2016.

[26] N. Kim, K. Lučivjanská, P. Molnár, and R. Villa, "Google searches and stock market activity: evidence from Norway," Finance Research Letters, vol. 28, pp. 208-220, 2019.

[27] H. A. Aalborg, P. Molnár, and J. E. de Vries, "What can explain the price, volatility and trading volume of Bitcoin?" Finance Research Letters, vol. 29, pp. 255-265, 2019.

[28] J. Li and J. Yu, "Investor attention, psychological anchors, and stock return predictability," Journal of Financial Economics, vol. 104, no. 2, pp. 401-419, 2012.

[29] D. Andrei and M. Hasler, "Investor attention and stock market volatility," Review of Financial Studies, vol. 28, no. 1, pp. 33-72, 2015.

[30] N. Vozlyublennaia, "Investor attention, index performance, and return predictability," Journal of Banking \& Finance, vol. 41, no. 4, pp. 17-35, 2014.

[31] X. Li, T. Qiu, G. Chen, L.-X. Zhong, and X.-F. Jiang, "Geography and distance effect on financial dynamics in the Chinese stock market," Physica A: Statistical Mechanics and Its Applications, vol. 457, pp. 109-116, 2016.

[32] R. Portes and H. Rey, "The determinants of cross-border equity flows," Journal of International Economics, vol. 65, no. 2, pp. 269-296, 2005.

[33] R. F. Engle, "Autoregressive conditional heteroscedasticity with estimates of the variance of United Kingdom inflation," Econometrica, vol. 50, no. 4, pp. 987-1008, 1982.

[34] P. H. Franses and D. Van Dijk, "Forecasting stock market volatility using (non-linear) garch models," Journal of Forecasting, vol. 15, no. 3, pp. 229-235, 1996.

[35] A. Q. Barbi and G. A. Prataviera, "Nonlinear dependencies on Brazilian equity network from mutual information minimum spanning trees," Physica A: Statistical Mechanics and Its Applications, vol. 523, pp. 876-885, 2019.

[36] M. Papapetrou and D. Kugiumtzis, "Tsallis conditional mutual information in investigating long range correlation in symbol sequences," Physica A: Statistical Mechanics and Its Applications, vol. 540, p. 123016, 2020.

[37] R. Steuer, J. Kurths, C. O. Daub, J. Weise, and J. Selbig, "The mutual information: detecting and evaluating dependencies between variables," Bioinformatics, vol. 18, no. 2, pp. 31-40, 2002.

[38] D. W. Scott, Multivariable Density Estimation: Theory, Practice, and Visualization, Wiley, New York, NY, USA, 1992.

[39] D. Andreia, R. Menezes, and D. A. Mendes, "Entropy-based independence test," Nonlinear Dynamics, vol. 44, no. 1-4, pp. 351-357, 2006.

[40] C. Granger and J.-L. Lin, "Using the mutual information coefficient to identify lags in nonlinear modelsficient to identify lags in nonlinear models," Journal of Time Series Analysis, vol. 15, no. 4, pp. 371-384, 1994.

[41] J. W. Kantelhardt, S. A. Zschiegner, E. Koscielny-Bunde, S. Havlin, A. Bunde, and H. E. Stanley, "Multifractal detrended fluctuation analysis of nonstationary time series," Physica A: Statistical Mechanics and Its Applications, vol. 316, no. 1-4, pp. 87-114, 2002.

[42] B. Podobnik, D. Horvatic, A. M. Petersen, and H. E. Stanley, "Cross-correlations between volume change and price change," Proceedings of the National Academy of Sciences, vol. 106, no. 52, pp. 22079-22084, 2009.
[43] W. X. Zhou, "Multifractal detrended cross-correlation analysis for two nonstationary signals," Physical Review E Statistical Nonlinear \& Soft Matter Physics, vol. 77, no. 6, p. 66211, 2008.

[44] S. Shadkhoo and G. R. Jafari, "Multifractal detrended crosscorrelation analysis of temporal and spatial seismic data," The European Physical Journal B, vol. 72, no. 4, pp. 679-683, 2009.

[45] G. F. Zebende, "DCCA cross-correlation coefficient: quantifying level of cross-correlation," Physica A: Statistical Mechanics and Its Applications, vol. 390, no. 4, pp. 614-618, 2011.

[46] X. Zhao, P. Shang, and J. Huang, "Several fundamental properties of DCCA cross-correlation coefficient," Fractals, vol. 25, no. 2, Article ID 1750017, 2017.

[47] U. Bashir, G. F. Zebende, Y. Yu, M. Hussain, A. Ali, and G. Abbas, "Differential market reactions to pre and post Brexit referendum," Physica A: Statistical Mechanics and Its Applications, vol. 5151, pp. 151-158, 2019.

[48] E. F. Guedes and G. F. Zebende, "DCCA cross-correlation coefficient with sliding windows approach," Physica A: Statistical Mechanics and Its Applications, vol. 527, Article ID 121286, 2019.

[49] B. Podobnik and H. E. Stanley, "Detrended cross-correlation analysis: a new method for analyzing two nonstationary time series," Physical Review Letters, vol. 100, no. 8, p. 84102, 2008.

[50] B. Podobnik, I. Grosse, D. Horvatić, S. Ilic, P. C. Ivanov, and H. E. Stanley, "Quantifying cross-correlations using local and global detrending approaches," The European Physical Journal $B$, vol. 71, no. 2, pp. 243-250, 2009.

[51] J. D. Hamilton and R. Susmel, "Autoregressive conditional heteroskedasticity and changes in regime," Journal of Econometrics, vol. 64, no. 1-2, pp. 307-333, 1994.

[52] F. Klaassen, "Improving GARCH volatility forecasts with regime-switching GARCH," Empirical Economics, vol. 27, no. 2, pp. 363-394, 2002.

[53] Y. Wang, Y. Wei, and C. Wu, "Cross-correlations between Chinese A-share and B-share markets," Physica A: Statistical Mechanics and Its Applications, vol. 389, no. 23, pp. 54685478, 2010. 